\title{
WORMHOLES IN SPACETIME AND THE CONSTANTS OF NATURE*
}

\author{
JOHN PRESKILL****** \\ California Institute of Technologv, Pasadena, CA 91125, USA
}

Received 24 November 1988

\begin{abstract}
The proposal that wormholes in spacetime cause the cosmological constant $A$ to vanish is reviewed, and its implications are studied. Wormholes also drive Newton's constant $G$ to the lowest possible value. The requirement that $G$ is at its minimum determines, in principle, all other constants of Nature. In practice, the values of fundamental constants other than $A$ cannot be predicted without a detailed knowledge of Planck-scale physics.
\end{abstract}

\section{Introduction}

A wormhole in spacetime is a gravitational quantum fluctuation that links two distantly separated spacetime points [1-3]. Although it is not yet clearly understood whether such fluctuations must be included in a sensible quantum theory of gravity, it is at least a plausible hypothesis that wormhole fluctuations occur, and it is interesting to contemplate the consequences of this hypothesis.

The euclidean path integral approach to quantum gravity [4-6] provides a formalism in which wormhole effects can be systematically discussed; among the four-dimensional geometries that contribute to the path integral are wormhole configurations in which two points on a smooth background spacetime are connected by a narrow tube, as in fig. 1. But even if the euclidean path integral as currently formulated does not provide an adequate description of quantum gravity, it is certainly possible that wormholes exist and have remarkable effects.

It is natural to wonder whether wormholes can induce an apparent failure of locality in the physics on the background spacetime, or, as Hawking [7] has advocated, an apparent loss of quantum coherence. Coleman, however, has argued persuasively that the physical effects of wormholes are quite different than we might

\footnotetext{
* This work supported in part by the U.S. Department of Encrgy under Contract No. DE-AC0381ER40050.

** NSF Presidential Young Investigator.

*** Bitnet address: Preskill@CALTECH.
} 


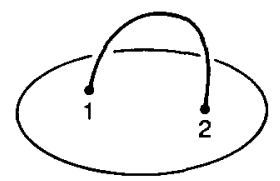

Fig. 1. A wormhole connects points 1 and 2 that are distantly separated on the background spacetime.

naively expect $[8,9]$. He concludes that, because of wormhole effects, the fundamental constants of Nature are afflicted by an intrinsic quantum indeterminacy; we must regard our universe as having been chosen at random from an ensemble of possible universes, each with different values of the fundamental constants. Quantum gravity, then, may threaten our ability, even in principle, to make precise predictions about how Nature behaves.

Having created this predicament, Coleman also, in a subsequent paper [10], suggested a means of escaping it ${ }^{\star}$. He argued that the probability distribution of possible universes is in fact a very sharply peaked function, so that it is overwhelmingly likely that a randomly selected universe will have values of the constants of Nature that are precisely determined. If correct, this claim restores in principle our hopes of predicting how Nature behaves. In practice, predictive power is reclaimed only if we can compute the values of the fundamental constant at which the sharp peak occurs. Coleman has computed one such constant; he predicts that the cosmological constant $\Lambda$ is exactly zero. This prediction is in accord with observation.

In this paper, I will examine whether Coleman's reasoning can be applied to determine other constants of Nature, aside from the cosmological constant ${ }^{\star \star}$. I will also address some challenges to the consistency of Coleman's arguments that one might raise. For example, Coleman assumed that wormholes have a characteristic "thickness" that is not much greater than the Planck length. One may wonder whether wormholes of arbitrarily large thickness can in fact contribute significantly to low-energy processes. Large wormholes, if their effects are unsuppressed, would be hard to reconcile with the well-tested successes of local field theory in describing low-energy physics $[20,16,18]$. One may also worry that assumptions similar to those that lead to the successful prediction $\Lambda=0$ will lead to unsuccessful predictions concerning the values of other fundamental constants $[15,16]$. Any such wrong prediction would cast doubt upon the foundation that underlies Coleman's solution to the cosmological constant problem.

The main conclusions of this paper are as follows: First of all, I argue that all of the constants of Nature do indeed have precisely determined values. That is, for each constant there is a "standard" value that is in principle calculable, and in a

\footnotetext{
${ }^{\star}$ Related ideas appeared in ref. [11].

$\star \star$ This issue and other related issues have also been addressed in refs. [12-19].
} 
randomly selected universe the probability is one that each constant assumes its standard value. Thus, the quantum indeterminacy that afflicts the fundamental constants turns out to be very mild. The standard value of the cosmological constant $\Lambda$ can be calculated; it is zero, as Coleman claimed. This result holds quite generally, and is not sensitive to the detailed properties of short-distance physics. But I argue that the standard values of all other fundamental constants are quite sensitive to the details of physics at the Planck scale; hence, no constants other than the cosmological constant can be explicitly calculated. This conclusion is rather disappointing, if not entirely surprising. To put it in a positive light, one might be encouraged that, because of our inability to extract other precise predictions, Coleman's beautiful explanation for $\Lambda=0$ has not been found to be inconsistent with anything that we know about Nature. Indeed, I will also propose a mechanism that can account naturally for the suppression of large wormholes.

Most of the analysis reported in this paper is premised on the claim that all of the constants of Nature other than the cosmological constant can be determined by requiring that Newton's gravitational constant $G$ must assume the smallest possible value $^{\star}$. The potential quantum indeterminacy of fundamental physics is evaded, because a randomly selected universe is overwhelmingly likely to have $G$ at its minimum. This criterion is sufficient to determine all of the fundamental constants because all contribute to $G$ through renormalization effects. The analysis consists of two main parts. In the first part, I study the effects of wormhole interactions, which were neglected in Coleman's original analysis. These interactions are essential in ensuring that $G$ actually has a nonzero minimum. They are also the key to understanding why the effects of large wormholes are suppressed. In the second part, the renormalization group method is applied to study the dependence of $G$ on the other fundamental constants. It is because the renormalization of $G$ is dominated by "nonuniversal" short-distance effects that we are unable in the end to make precise predictions about the values of the other constants.

The remainder of this paper is organized as follows: In sect. 2, the connection is derived between wormholes in spacetime and the potential quantum indeterminacy of the constants of Nature. As Coleman showed, one can "integrate out" the wormhole fluctuations and obtain an effective field theory with an explicit short-distance cutoff in which wormhole fluctuations no longer occur; the cutoff is the characteristic wormhole "thickness." The effects of the wormholes can then be incorporated into renormalization of the parameters of the effective theory. But there is another, more surprising, remnant of the wormhole fluctuations in low-energy physics. The effective field theory is actually a superposition of many "superselection" sectors that do not communicate with each other through any local physics.

\footnotetext{
* This criterion was previously suggested by Grinstein and Wise [15]. A different criterion was envisioned in refs. $[16,18]$; this accounts for some of the differing conclusions of refs. $[16,18]$ and the present paper.
} 
These sectors are labeled by a set of parameters, denoted by $\alpha$, that are presumably infinite in number. In each sector, the "bare" couplings that specify the effective field theory at the wormhole scale are functions of $\alpha$; this is the origin of the quantum indeterminacy of the fundamental constants.

The derivation presented here of this $\alpha$-dependence of the effective theory emphasizes the generality of the result. In particular, the argument does not rely at all on the semiclassical approximation invoked by Coleman. We will also see that the interpretation of $\alpha$ as the label of a coherent superposition of "baby universe" states, which played a central role in Coleman's discussion, is really peripheral to the argument, and may not even make sense beyond the semiclassical approximation.

In sect. 3, Coleman's explanation for the vanishing of the cosmological constant is reviewed. The superposition of $\alpha$-dependent effective theories is shown to be described by a probability distribution that is very sharply peaked at $A=0$. This argument requires some additional assumptions beyond those needed in sect. 2 . In particular, the argument seems to rely quite heavily on the validity of the euclidean path integral approach to quantum gravity .

In sect. 4, I argue that the probability distribution of the " $\alpha$-universes" strongly favors not only that $\Lambda$ vanish, but also that Newton's constant $G$ assume the smallest possible ( $\alpha$-dependent) value ${ }^{\star \star}$. Again, this argument seems to make essential use of the euclidean path integral formalism. I claim that the requirement that $G(\alpha)$ is at its minimum suffices to determine all of the $\alpha$ 's, and hence to determine all of the constants of Nature. The rest of the paper further explores the implications of this claim.

If we hope to actually determine the $\alpha$ 's by minimizing $G$, we must compute the function $G(\alpha)$. We can imagine carrying out such a calculation in two stages. In the first stage, we integrate out the wormhole fluctuations to obtain an effective theory with $\alpha$-dependent bare couplings; this effective theory is cut off at the wormhole mass scale $M_{\mathrm{w}}$. In the second stage, we allow the cutoff to "float" down from $M_{\mathrm{w}}$ into the far infrared; we thus obtain an expression for the renormalized $G$ that describes gravity at long distances in terms of the bare parameters of the effective theory. The first stage of this calculation is the subject of sect. 5 , and the second stage is the subject of sect. 6 .

If the mechanism proposed by Coleman for ensuring that $\Lambda=0$ is to apply to Nature, and if this mechanism requires $G(\alpha)$ to be at its minimum, then it is evidently necessary for $G(\alpha)$ to have a nonzero minimum. Otherwise $G$ will want to vanish, in conflict with observation. But in the approximation assumed by Coleman, in which wormholes are treated as dilute and the interactions among wormholes are

\footnotetext{
* The observation that $\Lambda=0$ is highly "probable" in euclidean quantum gravity had been made earlier by Hawking [21] and by Baum [22]. They, however, had not appreciated the crucial role of wormholes in making $\Lambda$ an adjustable quantity.

${ }^{\star}$ More precisely, $G(\alpha)$ is minimized as a function of $\alpha$ on that surface in $\alpha$-space where $A(\alpha)=0$.
} 
neglected, $G$ is not bounded away from zero. Thus, it is necessary to improve on this dilute approximation. The systematic corrections to the dilute approximation are described in sect. 5 . I indicate how wormhole interactions can generate a lower bound on $G$, and illustrate this possibility with a simple toy model.

The study of wormhole interactions will also lead me to propose a mechanism that can account for the suppression of the low-energy effects of large wormholes. This mechanism relies on the observation that the $\alpha$ 's are determined by the requirement that $G(\alpha)$ is at its minimum, and it arises due to the interactions of large wormholes with small wormholes. The mechanism has the unusual feature that the suppression of large wormholes cannot be understood in terms of long-distance physics alone. In this sense, it may be regarded as a violation of the principle that short-distance physics is effectively "decoupled" from long-distance physics.

In sect. 6, I describe how an effective theory of gravity is renormalized as the ultraviolet cutoff floats down from the wormhole scale toward the far infrared. I note that some nonperturbative properties of this renormalization can be extracted if I make the rather mild assumption that Einstein gravity has no nontrivial continuum limit. From these properties, I then infer the conditions that the $\alpha$-dependent bare theory at the wormhole scale must satisfy, in order that Coleman's derivation of $A=0$ be applicable. These conditions are found to be quite weak.

I then examine whether calculations of fundamental constants other than $\Lambda$ can be carried out in practice. I argue that the values of other constants cannot be precisely predicted without a detailed knowledge of physics at the wormhole scale (which is comparable to the Planck scale). This argument is based on straightforward power-counting of Feynman diagrams, which shows that the renormalization of $G$ is dominated by short-distance quantum fluctuations that are sensitive to the details of Planck-scale physics.

The analysis of renormalization effects leads to one rather surprising conclusion. I find that if an elementary scalar or fermion has an $\alpha$-dependent bare mass that can be adjusted at the wormhole scale, then the physical mass of this particle cannot be far below the wormhole scale, unless the physical mass vanishes exactly. Among other consequences, this observation implies that if Coleman's mechanism is to be compatible with the known features of particle physics, then we must insist that the light mass scales of particle physics (like the weak interaction scale) are determined dynamically. The weak interaction scale must not be determined by adjusting a bare mass parameter at the wormhole scale.

\section{The effects of wormholes}

A wormhole in spacetime is a gravitational quantum fluctuation that links two distantly separated spacetime points. Hence, it is natural to wonder whether wormholes can induce an apparent violation of locality. But Coleman [8] has argued 
persuasively that the physical effects of wormholes are quite different than we might naively expect. He concludes that, because of wormhole effects, the fundamental constants of Nature are afflicted with an intrinsic quantum indeterminacy. Quantum gravity, then, may threaten our ability, even in principle, to make precise predictions about how Nature behaves.

In this section, I will briefly review how this remarkable conclusion is reached, following closely a reformulation of Coleman's original argument due to Klebanov, Susskind, and Banks [16]. I include this section to establish notation, and also to make two important points. First, although Coleman originally presented his argument in the context of a semiclassical approximation, the validity of this approximation is not at all a necessary ingredient in the argument. This point is important because we will see when we apply Coleman's ideas to the cosmological constant problem that the semiclassical approximation used by Coleman cannot be justified. Second, while the notion of a "baby universe" associated with a wormhole played a central role in Coleman's discussion, this notion is actually peripheral to the argument, and may not make sense beyond the semiclassical approximation.

The basic assumptions underlying Coleman's analysis are that wormholes exist, and that the wormholes have two essential properties: (i) Wormholes have a characteristic "thickness" $R_{\mathrm{w}}$. This thickness is presumably of order the Planck length $M_{\mathrm{P}}^{-1}$, the characteristic length scale of gravitational quantum fluctuations. We assume that wormholes much thicker than $R_{\mathrm{w}}$ are rare and can be neglected ${ }^{\star}$. (ii) Wormholes have no characteristic length. We assume that the wormhole is completely indifferent to the separation between its two ends in the background spacetime; it takes a short cut.

It follows from assumption (i) that, for the purpose of discussing physics at energies well below $R_{\mathrm{w}}^{-1}$, wormholes may be integrated out; their effects may be incorporated into an effective field theory in which wormhole fluctuations no longer occur. In this low-energy effective theory, the contribution to a quantum mechanical expectation value due to a wormhole that connects the spacetime points $x_{1}$ and $x_{2}$ can be represented by an expansion in local operators at $x_{1}$ and $x_{2}$, of the form

$$
\sum_{a b} \Delta_{a b} \mathcal{O}_{a}\left(x_{1}\right) \mathcal{O}_{b}\left(x_{2}\right)
$$

Here, the $\hat{O}_{a}$ 's are a suitable basis for the gauge-invariant local operators, and $\Delta$ is a real symmetric matrix. By the assumption (ii) above, $\Delta$ is independent of $x_{1}$ and $x_{2}$. The bilocal expansion eq. (2.1) is understood to be valid when the separation between $x_{1}$ and $x_{2}$ is large compared to $R_{\mathrm{w}}$.

\footnotetext{
${ }^{\star}$ We will examine more closely in sect. 5 the justification of this assumption.
} 
After an integration over all possible positions of the wormhole ends on the background spacetime, the contribution eq. (2.1) becomes ${ }^{\star}$

$$
C \equiv \frac{1}{2} \int \mathrm{d}^{4} x_{1} \sqrt{g_{1}} \int \mathrm{d}^{4} x_{2} \sqrt{g_{2}} \sum_{a b} \Delta_{a b} \mathcal{O}_{a}\left(x_{1}\right) \mathcal{O}_{b}\left(x_{2}\right)
$$

Now, in treating the effect of a configuration of $N$ wormholes, let us imagine that the wormholes are sufficiently "dilute" that they may be considered to be independent of one another. The $N$-wormhole contribution then factorizes and can be expressed as $C^{N} / N$ !, where the $1 / N$ ! compensates for overcounting of identical wormholes. (In sect. 5 we will see how this dilute approximation can be systematically improved.)

The contribution due to an arbitrary wormhole configuration in the dilute limit can now be obtained by summing over $N$. The sum exponentiates, and can be expressed as a gaussian integral,

$$
\mathrm{e}^{C}=\int(\mathrm{d} \alpha) \exp \left(-\frac{1}{2} \sum_{a, b} \alpha_{a}\left(\Delta^{-1}\right)_{a b} \alpha_{b}\right) \exp \left(\sum_{a} \alpha_{a} \int \mathrm{d}^{4} x \sqrt{g} \mathcal{O}_{a}(x)\right)
$$

We see that, in the dilute approximation, the process of integrating out wormholes induces an $\alpha$-dependent shift in the local (euclidean) effective action,

$$
\delta S(\alpha)=-\sum_{a} \alpha_{a} \int \mathrm{d}^{4} x \sqrt{g} \mathcal{O}_{a}(x)
$$

Quantum mechanical expectation values in the effective theory are expressed as integrals over $\alpha$ of expectation values computed in universes with $\alpha$-dependent couplings ${ }^{\star}$. The various $\alpha$-universes are weighted by a probability distribution

$$
P(\alpha)=\exp \left(-\frac{1}{2} \sum_{a, b} \alpha_{a}\left(\Delta^{-1}\right)_{a b} \alpha_{b}\right)
$$

However, our physical observations are actually carried out in a universe with a fixed value of the $\alpha_{a}$ 's, for we in principle can measure the constants of Nature to arbitrary accuracy.

The loss of quantum coherence declaimed by Hawking [7] may be interpreted as a loss of information about the other $\alpha$-universes that occur in the superposition eq. (2.3). Because physics is done with all $\alpha_{a}$ 's fixed, this loss of coherence is not

\footnotetext{
* We neglect a small error due to the breakdown of the bilocal expansion for $x_{1} \sim x_{2}$.

$\star \star$ The number of independent $\alpha$ 's is the same as the number of nonzero eigenvalues of the matrix $\Delta$ in eq. (2.1). "Generically", this is the same as the number of gauge-invariant local operators; that is, it is infinite.
} 
accessible to any observer. Instead of a failure of quantum mechanics or of locality, wormhole effects introduce into physics a fundamental quantum indeterminacy of the constants of Nature. Wormholes threaten to inhibit our ability to predict with certainty the constants of Nature on the basis of fundamental theory.

That the $\alpha$-distribution $P(\alpha)$ is gaussian, and that the shift of the effective action $\delta S(\alpha)$ is linear in $\alpha$, are artifacts of the dilute approximation that we have made. Corrections to this approximation will be discussed in sect. 5 . But the conclusion that the effects of wormholes can be incorporated into an $\alpha$-dependent renormalization of the effective action seems to be much more general. I believe that this conclusion follows from just the two assumptions stated above (subject to some caveats that will be mentioned later).

The above reformulation of Coleman's argument was previously presented in ref. [16]. It differs from Coleman's original presentation [8] in two significant respects. First, Coleman's argument was carried out within the context of the semiclassical approximation, in which the only wormhole configurations that contribute to the euclidean path integral are solutions to the euclidean equations of motion (stationary points of the euclidean action) and small fluctuations about such solutions. The above argument is more satisfying because it makes no reference to the semiclassical approximation. This point is important, because we will see in sect. 3 that, if we are to invoke Coleman's arguments to solve the cosmological constant problem, then the semiclassical approximation cannot be justified. (We have, so far, regarded the wormholes as dilute, but our conclusions survive even when the dilute approximation is abandoned.)

The second respect in which our formulation differs from Coleman's original argument is that Coleman attaches to $\alpha$ an interpretation that was eschewed above. Coleman includes in his analysis, in addition to wormholes attached to the background spacetime, "semiwormholes" that connect the background spacetime to a tiny closed three-geometry, or "baby universe." He is thus able to interpret $\alpha$ as the label of a coherent superposition of baby universe states. As we have seen, this interpretation is not essential in the derivation of the main result, that wormhole effects can be absorbed into an $\alpha$-dependent effective action. This is a good thing, because it seems that the notion of a baby universe, or at least the connection between baby universes and the $\alpha$ 's associated with wormhole physics, makes sense only within the semiclassical approximation.

Although this is somewhat off the main line of the argument in this paper, I will describe in the remainder of this section how the connection between $\alpha$ 's and baby universes can be established. This discussion will clarify why the semiclassical approximation appears to be essential.

Let the index $i$ label the states of a basis for the baby universe states. We may define $S_{i j}$ as the amplitude for the baby universe state $i$ to evolve to the baby universe state $j$ determined, for example, by a euclidean path integral (fig. 2). We may also consider the amplitude for a semiwormhole to connect baby universe $i$ to 


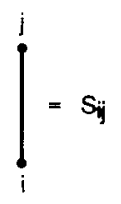

Fig. 2. The amplitude for baby universe state $i$ to evolve to baby universe state $j$.

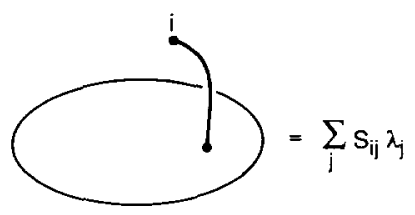

Fig. 3. The amplitude for baby universe state $i$ to connect via a semiwormhole to the background spacetime.

a background spacetime (fig. 3); quantities $\lambda_{j}$ can be defined (if the matrix $S$ is invertible) so that this amplitude is denoted

$$
\sum_{j} S_{i j} \lambda_{j}
$$

In this expression, we may think of $S$ as a baby universe "propagator," and $\lambda_{j}$ as the strength of the "coupling" of baby universe $j$ to the background spacetime.

So far this is just definitions. But now we make the nontrivial assumption that the amplitude for a wormhole to attach to the background spacetime is determined by the baby universe propagator and the semiwormhole amplitude to be

$$
\sum_{i j} \lambda_{i} S_{i j} \lambda_{j}
$$

Heuristically, then, the wormhole amplitude (fig. 4) obeys a "cutting rule" or "completeness relation," denoted schematically in fig. 5; it can be reproduced by a sum over baby universe "intermediate states."

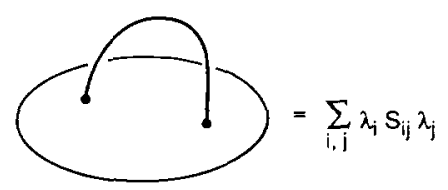

Fig. 4. The amplitude for a wormhole to attach to the background spacetime. 


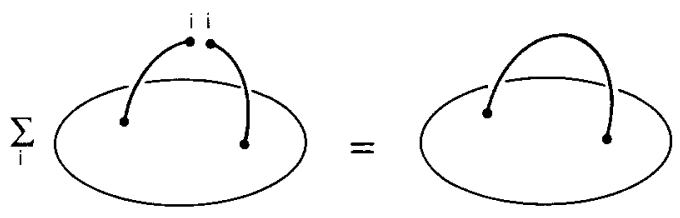

Fig. 5. The baby universe completeness relation.

In the semiclassical approximation, such a completeness relation is satisfied, because there is a natural way to associate a baby universe with a wormhole. The baby universe is the three-geometry obtained when we slice the classical wormhole solution through its midpoint $[2,8,9]$. But there is no unambiguous way of associating a general wormhole configuration (that is not a classical solution) with a baby universe, and I see no reason to expect that there exists a basis for the baby universe states such that the identity indicated in fig. 5 is satisfied, except in the semiclassical approximation.

This completeness relation provides the logical connection that is needed to relate wormhole physics to baby universes. To establish this relationship, assume the validity of the completeness relation and sum over wormhole and semiwormhole configurations. (A typical configuration is shown in fig. 6.) In the dilute approximation the sum exponentiates, and we have

$$
Z[J]=\exp \left(\sum_{i, j}\left(\frac{1}{2} J_{i} S_{i j} J_{j}+J_{i} S_{i j} \lambda_{j}+\frac{1}{2} \lambda_{i} S_{i j} \lambda_{j}\right)\right)
$$

where $J_{i}$ is a source that couples to the baby universe $i$. This generating function can be expressed as a gaussian integral

$$
Z[J]=\int(\mathrm{d} \alpha) \exp \left(-\frac{1}{2} \sum_{i, j} \alpha_{i}\left(S^{-1}\right)_{i j} \alpha_{j}+\sum_{i} \alpha_{i}\left(J_{i}+\lambda_{i}\right)\right)
$$

Now it is convenient to redefine our basis for the baby universe states so that $S_{i j}$ becomes $\delta_{i j}$. (This is a conventional "renormalization" of the baby universe wave

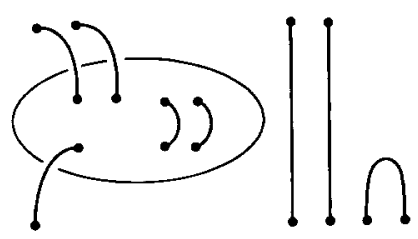

Fig. 6. A typical configuration of wormholes and semiwormholes. 
functions.) In this new basis, the sources and couplings become

$$
\begin{aligned}
& J_{i}^{\prime}=\left(S^{1 / 2}\right)_{i j} J_{j}, \\
& \lambda_{i}^{\prime}=\left(S^{1 / 2}\right)_{i j} \lambda_{j},
\end{aligned}
$$

and after a change of variable in the integral, our expression for the generating function becomes

$$
Z\left[J^{\prime}\right]=\prod_{i} \int\left(\mathrm{d} \alpha^{\prime}\right) \exp \left[-\frac{1}{2} \alpha_{i}^{\prime} \alpha_{i}^{\prime}+\alpha_{i}^{\prime}\left(J_{i}^{\prime}+\lambda_{i}^{\prime}\right)\right]
$$

up to a normalization factor, independent of $J$ and $\lambda$, that can be absorbed into the integration measure.

Wick's theorem tells us that

$$
\int(\mathrm{d} \alpha) \mathrm{e}^{-(1 / 2) \alpha^{2}} \alpha^{n}=\left\langle 0\left|\left(a+a^{\dagger}\right)^{n}\right| 0\right\rangle
$$

where $a, a^{\dagger}$ are conventionally normalized annihilation and creation operators satisfying $\left[a, a^{\dagger}\right]=1$. We therefore have

$$
\begin{aligned}
Z\left[J^{\prime}\right] & =\prod_{i}\left\langle 0\left|\exp \left[\left(\lambda_{i}^{\prime}+J_{i}^{\prime}\right)\left(a_{i}+a_{i}^{\dagger}\right)\right]\right| 0\right\rangle \\
& =\prod_{i}\left[\mathrm{e}^{(1 / 2) J_{\text {in }, i}^{2}} \mathrm{e}^{(1 / 2) J_{\text {out }, i}^{2}}\left\langle 0\left|\mathrm{e}^{J_{\text {out }, i} a_{i}} \mathrm{e}^{\lambda_{i}^{\prime}\left(a_{i}+a_{i}^{\dagger}\right)} \mathrm{e}^{J_{\text {in }, i} a_{i}^{\dagger}}\right| 0\right\rangle\right]
\end{aligned}
$$

In the second equality in eq. (2.11) we have written $J_{i}^{\prime}=J_{\text {in, } i}+J_{\text {out, },}$, in order to distinguish "incoming" and "outgoing" baby universe states, and have invoked the identity $\mathrm{e}^{A+B}=\mathrm{e}^{A} \mathrm{e}^{B} \mathrm{e}^{-(1 / 2)[A, B]}$ that is satisfied by operators $A$ and $B$ that commute with $[A, B]$. The prefactor $\mathrm{e}^{(1 / 2) J_{\text {in, } i}^{2}} \mathrm{e}^{(1 / 2) J_{\text {oul }, i}^{2}}$ in this identity is associated with incoming (outgoing) states that propagate to other incoming (outgoing) states (fig. 7). We did not intend to include such processes. (It is inappropriate to include them in the semiclassical approximation.) So we suppress the prefactor and obtain

$$
\left.\left\langle m_{1}, m_{2}, \ldots, \text { out }\right| n_{1}, n_{2}, \ldots, \text { in }\right\rangle=\prod_{i}\left\langle m_{i}\left|\mathrm{e}^{\lambda_{i}^{\prime}\left(a_{i}+a_{i}^{\dagger}\right)}\right| n_{i}\right\rangle
$$

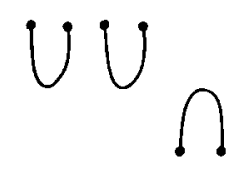

Fig. 7. Processes that are excluded from the wormhole sum. 
This result was derived by Coleman [8] and by Strominger and Giddings [9]. It shows that $\alpha_{i}^{\prime}$ can be interpreted as the operator $a_{i}+a_{i}^{\dagger}$ acting on baby universe states of type $i$, and that physics at fixed $\alpha_{i}^{\prime}$ is physics in a "condensate" of baby universes, an eigenstate of the "position" operator of the baby universe "oscillator." But to justify it, we needed to assume not only the dilute approximation, but also the baby universe completeness relation (fig. 5).

Thus, the identification of $\alpha$ as the label of a baby universe state may make sense only within the semiclassical approximation. That is why I emphasize that Coleman's main conclusion, that wormhole effects induce an $\alpha$-dependent renormalization of the local effective action, does not rely on this interpretation of the $\alpha$ 's.

\section{The cosmological constant}

The discussion of the previous section indicates that, because of wormhole effects, the constants of Nature are afflicted with an inescapable indeterminacy. Our universe has been chosen at random from an ensemble of possible universes, all with different values of the $\alpha$ 's, and hence of the fundamental constants. Wormholes in spacetime threaten to render unachievable the greatest ambition of physics - to predict the constants of Nature as consequences of a complete fundamental theory.

Having created this predicament, Coleman [10] also suggested a means of escaping it. He argued that the distribution of possible universes is a very sharply peaked function of the $\alpha$ 's. Thus, it is overwhelmingly likely that a randomly selected universe will have values of the constants of Nature that are precisely known.

This claim, if correct, restores in principle our hopes of predicting how Nature behaves. In practice, predictive power is reclaimed only if we can compute the values of the fundamental constants at which the sharp peak occurs. Coleman has computed one such constant; he predicts that the cosmological constant is exactly zero. This prediction is confirmed by observation.

Our main objective in this paper is to examine how Coleman's reasoning can be applied to determine the other constants of Nature, aside from the cosmological constant. But as a prelude to that main topic, I will review in this section how the cosmological constant is calculated.

This calculation relies more heavily than the results of the previous section of the specific features of the euclidean quantum gravity formalism. To begin, recall that in this formalism $[4-6,10]$, the expectation value of a gauge-invariant local observable $\mathcal{O}$ is expressed as

$$
\langle\mathcal{O}\rangle=N \int(\mathrm{d} g) \mathrm{e}^{-S[g]} \mathcal{O} ;
$$

here $S[g]$ is the euclidean action, $N$ is a normalization factor, and the path integral 


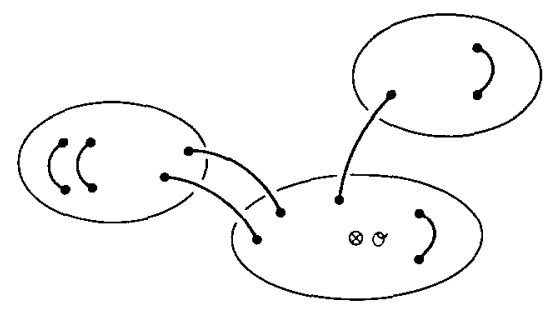

Fig. 8. A connected geometry that becomes disconnected after wormholes are integrated out. The operator $\mathcal{O}$ whose expectation value is to be evaluated acts on one of the connected components.

is over four-dimensional geometries that are closed and connected ${ }^{\star}$. (The integration over fields other than the metric has not been explicitly indicated, but is understood.) We assume as in sect. 2 that this path integral includes a sum over configurations in which narrow wormholes link distantly separated points on a smooth background spacetime, and we imagine "integrating" out the wormholes and incorporating their effects into a local effective action for the background spacetime. But now we note that the connected closed manifolds include geometries in which many distinct large smooth manifolds are linked together by narrow wormholes. Thus, when we integrate out wormholes, a typical connected closed manifold may yield many disconnected components (fig. 8). Integrating out wormholes, then, affects the euclidean path integral in two ways. As we already saw in sect. 2, expectation values become expressed in the effective theory as integrals over the $\alpha$ parameters of expectation values computed in various universes with $\alpha$-dependent couplings. But furthermore, the sum over connected closed manifolds in the underlying theory becomes a sum over all closed manifolds, including disconnected ones, in the effective theory.

Each disconnected manifold can be factored into a connected component on which the operator $\mathcal{O}$ acts, and a remainder on which no local operator acts. Since the sum over all closed four-dimensional geometries can be written as the exponential of a sum over all connected closed four-geometries, the expression for the expectation value of $\mathcal{O}$ may be written in the effective theory as

$$
\langle\mathcal{O}\rangle=N \int(\mathrm{d} \alpha) P(\alpha) \exp \left(\int\left(\mathrm{d} g^{\prime}\right) \mathrm{e}^{-S\left[g^{\prime} \cdot \alpha\right]}\right) \int(\mathrm{d} g) \mathrm{e}^{-S[g \cdot \alpha]} \mathcal{O}
$$

where again the integrals over $g$ and $g^{\prime}$ denote sums over connected closed four-geometries, but with wormholes now excluded. The distribution $P(\alpha)$ is that considered in sect. 2 , which would be gaussian in the dilute approximation. But we

* In this discussion, the Hartle-Hawking boundary condition [6] has been implicitly adopted. The results are not sensitively dependent on this choice. 
now see that the measure for the integration over $\alpha$ contains the additional factor

$$
Q(\alpha) \equiv \exp \left(\int(\mathrm{d} g) \mathrm{e}^{-S[g, \alpha]}\right)
$$

It is Coleman's proposal that this factor is a sharply peaked function of the $\alpha$ 's; since $P(\alpha)$ is presumably a smooth function, the factor $Q(\alpha)$ determines where the support of the $\alpha$-distribution lies.

To compute the factor $Q(\alpha)$ in eq. (3.3), Coleman makes a further hypothesis that the sum over connected closed four-geometries is dominated by large smooth geometries with small curvature (in Planck units). The idea is that by integrating out wormholes we have satiated the desire of a typical four-geometry to crinkle up on the Planck scale. This assumption is in the same spirit as, though logically independent of, the assumption that it makes sense to integrate out wormholes at all. Surely, given the current status of our understanding of quantum gravity, both assumptions must be regarded as unproved working hypotheses.

Anyway, given that the integral $\int(\mathrm{dg}) \mathrm{e}^{-S[g, \alpha]}$ is dominated by large smooth geometries, we may compute it by the following procedure: Recall that $S[g, \alpha]$ is the action of an effective theory with a cutoff of order $M_{\mathrm{w}} \sim R_{\mathrm{w}}^{-1}$, the characteristic mass scale of the wormhole fluctuations. Imagine allowing this cutoff to float down to a mass scale $M \ll M_{w}$; we integrate out all quantum fluctuations with wave number $k>M$, and incorporate the effects of these fluctuations into a renormalized effective action $S_{M}[g, \alpha]$. This renormalized effective action can be expanded in operators that are local on the distance scale $M^{-1}$ [23].

For $M \ll M_{\mathrm{w}}$, the loop corrections in the effective theory with cutoff $M$, and the effects of higher dimension operators in $S_{M}$, are suppressed by powers of $M / M_{\mathrm{w}}$. Because loop corrections are small, the path integral of the effective theory can be evaluated to good accuracy semiclassically, with the result

$$
\int(\mathrm{d} g)_{M} \mathrm{e}^{-S_{M}[g, \alpha]}=\mathrm{e}^{-S_{M}[\bar{g}, \alpha]},
$$

where $\bar{g}$ is the four-geometry at which $S_{M}$ is stationary. If this stationary point is a large smooth geometry with volume of order $R^{4}$, we improve on the semiclassical approximation by allowing the cutoff to float down to $M \sim R^{-1}$. Then all fluctuations at wavelengths less than $R$ have been absorbed into the renormalization of the effective action, and fluctuations on larger wavelengths are absent because the volume acts as an infrared cutoff ${ }^{\star}$.

\footnotetext{
* I have described the procedure in terms of a floating cutoff in order to emphasize that the action may always be regarded as effectively local and also that infrared divergent loop integrals need not be encountered; they arise only as $R \rightarrow \infty$ or $M \rightarrow 0$.
} 
Of course, it was a bit disingenuous to say above that $\bar{g}$ is the stationary point of $S_{M}$; we really want $\bar{g}$ to minimize $S_{M}$. It is well known that the stationary points of the euclidean action of gravity are not even local minima; we can always perform a conformal transformation on the metric that lowers the action. In order that the above discussion apply I must assume that there is some correct procedure for dealing with the conformal fluctuations, and that when this procedure is invoked, conformal fluctuations can be integrated out and absorbed into a renormalized effective action.

The effective action $S_{M}$ can be expanded in terms of local operators, and, for $M \ll M_{\mathrm{w}}$, operators with $n$ derivatives acting on the metric have effects that are suppressed by the factor $\left(M / M_{\mathrm{w}}\right)^{n}$. This expansion has the form ${ }^{\star}$

$$
S_{M}[g]=\frac{1}{16 \pi G} \int \mathrm{d}^{4} x \sqrt{g}(2 A-R+\ldots)
$$

where terms with more than two derivatives have been neglected; the parameters $G$ and $\Lambda$ have implicit dependence on $M_{\mathrm{w}}, M$, and $\alpha$. At the stationary points of $S_{M}$, the metric obeys the euclidean Einstein equation

$$
R_{\mu \nu}=\Lambda g_{\mu \nu},
$$

and the action is

$$
S_{M}[\bar{g}]=-\frac{\Lambda}{8 \pi G} \int \mathrm{d}^{4} x \sqrt{g}=-\frac{\Lambda}{8 \pi G} V
$$

where $V$ is the volume of the euclidean spacetime. For $\Lambda>0$, the solution of eq. (3.6) with maximal volume is known to be a four-sphere with

$$
V=24 \pi^{2} / \Lambda^{2}
$$

Hence the factor eq. (3.3) is found to be

$$
Q(\alpha)=\exp \left(\mathrm{e}^{-S_{M}[\bar{g} \cdot \alpha]}\right)=\exp [\exp (3 \pi / G \Lambda)],
$$

for $\Lambda>0$. Here $\Lambda$ is the cosmological constant renormalized at the scale $M \sim V^{-1 / 4}$, with $V$ given by eq. (3.8).

Eq. (3.9) is the sharp peak at $\Lambda=0$ found by Coleman. $\Lambda$ is a function of $\alpha$, and eq. (3.9) together with eq. (3.2) tells us that it is overwhelmingly likely that a universe selected at random will have a value of $\alpha$ such that $\Lambda=0$, assuming that $\Lambda$ vanishes for some choice of $\alpha$. We note that the quantity that has been found to

* Massive fields (heavier than $M$ ) have been integrated out, and light fields have been set equal to values that minimize $S_{M}$, so $S_{M}$ is expressed as a functional of the metric only. 
vanish is the renormalized $\Lambda$ that one would actually measure in a large smooth universe; it includes the contributions from the fluctuations of all quantum fields. We also note that it was safe to neglect the higher dimension operators in eq. (3.5); these give contributions to $S_{M}[\bar{g}]$ that are independent of $\Lambda$ for $\Lambda$ small, and do not modify the $\Lambda=0$ singularity in eq. (3.9).

(The procedure that we have used to calculate $Q(\alpha)$ may make sense for $\Lambda>0$, but it is ill-defined for $\Lambda<0$. For $\Lambda<0$, the best approach may be to analytically continue eq. (3.9) from positive values of $\Lambda$ to negative values. Our implicit assumption that $G$ is non-negative will be defended in sect. 6.)

It may be worthwhile to comment on the use of "semiclassical" reasoning in this derivation. We treated gravity semiclassically in the sense that we argued that loop effects are small when the floating ultraviolet cutoff $M$ is much less than the cutoff $M_{\mathrm{w}}$ of the bare theory (and in fact comparable to the infrared cutoff $V^{-1 / 4}$ ). But we certainly did not require that the renormalization of $S$ as the cutoff floats from $M_{\mathrm{w}}$ to $M$ be susceptible to a perturbative treatment. Indeed, it may be a misstatement to say that the path integral is dominated by large smooth geometries, for we do not mean to exclude the possibility that the effective action is strongly renormalized by quantum fluctuations at scales just below $M_{\mathrm{w}}$. Nor, of course, have we required that the process of integrating out wormholes, which generated the theory with cutoff $M_{\mathrm{w}}$, be accurately described by the semiclassical approximation.

The observation that we made in sect. 2 - that the notion of a baby universe makes sense only within the context of an untrustworthy approximation - has a consequence that should be mentioned now. We have noted that a "superselection rule" applies to wormhole physics; universes with different values of $\alpha$ cannot communicate with each other through any local physical process. But Coleman's explanation for $\Lambda=0$ can be invoked only if we consider a superposition of these $\alpha$-universes as in eq. (3.2). One might object to such a superposition, citing the analogy with the $\theta$-vacua of QCD. It would be perverse to say that the ground state of QCD is a superposition of $\theta$-vacua. Why shouldn't we take the same attitude toward a superposition of $\alpha$-universes?

The key distinction between $\theta$-vacua and $\alpha$-universes, I suspect, is that the $\alpha$ superselection rule is an approximate rule that holds only in the low-energy limit. It would have been easier to imagine that the $\alpha$ superselection rule were exact if the interpretation of $\alpha$ as the label of a baby universe were also exact. If this interpretation fails, it is hard to see how $\alpha$-sectors can be identified in sub-Planck scale physics. This, perhaps, is the proper way to interpret Coleman's claim [10] that an a priori distribution in $\alpha$ with support at a single point in $\alpha$-space would be "unnatural."

The argument in this section evidently required stronger assumptions than the discussion in sect. 2 . Here we apparently needed to take more seriously than in sect. 2 the proposal that expectation values can be evaluated as euclidean path integrals (and that a sensible prescription exists for dealing with conformal fluctuations). 
And we further assumed that, after wormholes are integrated out, the path integral is dominated by large smooth geometries. That these assumptions lead to the exciting conclusion that the cosmological constant vanishes already provides sufficient motivation for exploring their further consequences. But it is obviously an urgent matter to understand better the justification for these assumptions, if indeed any can be found.

\section{Newton's constant}

We have emphasized that the factor $Q(\alpha)$ in the measure for the integration over $\alpha$-universes overwhelmingly favors $A=0$, if the renormalized cosmological constant can vanish for some value of $\alpha$. This measure factor has a further consequence; Newton's constant $G$ wants to assume the smallest possible value, preferably zero ${ }^{\star}$. In the remainder of this paper, we will explore the consequences of this tendency of $G$ to seek its minimum allowed value.

One consequence is immediate: the renormalized $G$ as a function of $\alpha$ must be bounded away from zero

$$
G(\alpha) \geqslant G_{\min }>0
$$

Otherwise, the same assumptions that lead to the successful prediction $\Lambda=0$ will also lead to the unsuccessful prediction $G=0$. (A gravitational interaction is observed in our universe.)

One might object, at first, to the assertion that the probability distribution

$$
Q(\alpha) \sim \exp [\exp (3 \pi / G \Lambda)]
$$

is capable of determining both $G$ and $\Lambda$. This claim sounds strange, because $Q(\alpha)$ is a function of only the product $G \Lambda$. Thus, one might argue, the very sharp peak in $Q(\alpha)$ occurs at $G A=0$, and $Q(\alpha)$ is completely indifferent to the value of $G$ once $A$ has assumed the value $\Lambda=0$.

Indeed, to extract from eq. (4.2) a probability distribution on the surface $G \Lambda=0$ (where $Q(\alpha)$ is singular), we must specify a preferred way of approaching this surface. I will argue below that the correct procedure is to evaluate $Q(\alpha)$ on surfaces of constant $\Lambda$, and then take the limit $\Lambda \rightarrow 0$. If $\Lambda$ assumes a very small positive value, $Q(\alpha)$ strongly favors that $G^{-1}$ increase. This tendency of $G^{-1}$ to increase is not suddenly lost when $\Lambda$ is exactly zero.

We claim, then, that the support of the distribution $Q(\alpha)$ will lie at that point (or those points) in $\alpha$-space where $G^{-1}(\alpha)$ assumes its maximal value on the surface defined by $\Lambda(\alpha)=0$. There is another objection that one could raise against this claim. One might question whether the requirement that $G^{-1}$ assumes its maximum

\footnotetext{
^ This point was stressed previously by Grinstein and Wise [15].
} 
has any physical content. Since $G^{-1}$ is a dimensional quantity, the argument might go, its value of course depends on the units in which we choose to express it. Nothing prevents us from choosing our unit of mass to be $G^{-1 / 2}$. Then $G^{-1}$ is one, and it makes no sense to "maximize" it.

This argument is also misleading, for several reasons. First of all, the distribution $Q(\alpha)$ quantifies the relative probabilities of different "theories" with different values of the constants of Nature. When two theories are compared, we should express the physical constants of both in the same units; it is valid, for example, to say that $G$ in theory $\mathrm{A}$ is smaller than $G$ in theory B. In fact, all of the theories that we are comparing share the same $\alpha$-independent ultraviolet cutoff, the wormhole scale $M_{\mathrm{w}}$. Thus, when we compare different theories, we may express all mass scales (like $G^{-1 / 2}$ ) as dimensionless quantities, in units of $M_{\mathrm{w}}$. Most important, the criterion that $G(\alpha) M_{\mathrm{w}}^{2}$ is at its minimum has consequences that (in principle) relate to experimentally accessible low-energy physics. Indeed, we will argue that this criterion actually fixes all of the dimensionless quantities that specify the properties of low-energy physics.

To support the claim that $Q(\alpha)$ should be evaluated on the surface $G \Lambda=0$ as a $\Lambda \rightarrow 0$ limit, we will regulate the very singular dependence of $Q(\alpha)$ on $\Lambda(\alpha)$ by introducing an infrared cutoff into the calculation of $Q(\alpha)$. The point is that $\Lambda$ controls the volume of the four-geometry that dominates the path integral ${ }^{\star}$.

Imagine that we repeat the calculation of $Q(\alpha)$ in sect. 3, but that the closed four-geometries that contribute to the path integral eq. (3.3) are now restricted to geometries with volume $V$ less than a maximum volume $V_{\max } ; V_{\max }$, then, is our infrared cutoff. We assume again that the path integral is dominated by the four-sphere geometry. Then we have

$$
Q(\alpha) \sim \exp \left[\exp \left(-S_{0}\right)\right]
$$

where $S_{0}$ is the minimal action of a four-sphere that satisfies the volume constraint $V \leqslant V_{\max }$.

For a four-sphere of radius $R$, the action eq. (3.5) becomes ${ }^{\star \star}$

$$
S(R)=\frac{1}{16 \pi G} \Omega\left(2 \Lambda R^{4}-12 R^{2}+\ldots\right),
$$

where $\Omega=8 \pi^{2} / 3$ is the volume of the unit four-sphere. The minimum ${ }^{\star \star \star}$ of $S(R)$

* We choose to regulate $Q(\alpha)$ by means of an infrared cutoff because such a regulator can be formulated in the bare theory, without any reference to $\alpha$. Since it is our desire to extract an unambiguous function of $\alpha$ on the surface $G A=0$, it seems sensible to describe the approach to this surface in a manner that does not introduce implicit $\alpha$-dependence.

* For sufficiently large $R, A$ and $G$ may be taken to be $R$-independent constants, because they "stop running" in the infrared limit. This will be explained at greater length in sect. 6 .

$\star \star \star$ As in sect. 3, this minimum exists only if $A>0$. For $A<0, Q(\alpha)$ may be defined by analytic continuation in $A$. 


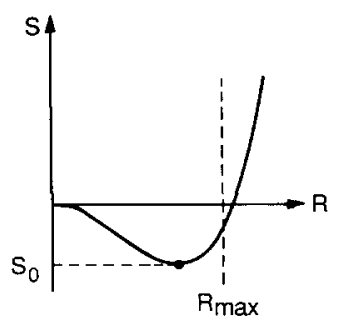

(a)

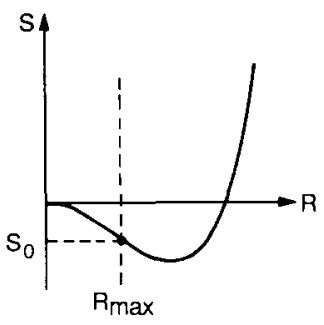

(b)

Fig. 9. The action $S$ for a four-sphere of radius $R$, with $A>0$. The minimum $S_{0}$ of the action is indicated (a) for $A \geqslant 3 R_{\max }^{2}$ and (b) for $A \leqslant 3 R_{\max }^{-2}$.

occurs at $R^{2}=3 \Lambda^{-1}$ for $\Lambda \geqslant 3 R_{\max }^{-2}$ and at $R^{2}=R_{\max }^{2}$ for $\Lambda \leqslant 3 R_{\max }^{-2}$, where $V_{\max }=\Omega R_{\max }^{4}$ (see fig. 9). Therefore, the minimum of the action is

$$
\begin{array}{ll}
S_{0}=-3 \pi / G \Lambda, & \Lambda \geqslant 3 R_{\max }^{-2}, \\
S_{0}=-(3 \pi / G \Lambda)\left[2\left(\frac{1}{3} \Lambda R_{\max }^{2}\right)-\left(\frac{1}{3} \Lambda R_{\max }^{2}\right)^{2}\right], & 0<\Lambda \leqslant 3 R_{\max }^{-2} .
\end{array}
$$

The typical value of $\Lambda$, then, in the distribution $Q(\alpha)$ is

$$
\Lambda \sim R_{\max }^{-2}
$$

which is independent of $G$. As the infrared cutoff is removed, $Q(\alpha)$ becomes very sharply peaked at $\Lambda=0$.

Now suppose that both $\Lambda$ and $G$ are functions of the $\alpha$ 's. We wish to minimize $S_{0}$ in eq. (4.5) as a function of the $\alpha$ 's. To accomplish this, we may find the minimum of $G(\alpha)$ on each $\Lambda(\alpha)=$ constant surface, and then subsequently minimize with respect to $\Lambda(\alpha)$. Obviously if $G(\alpha)=0$ for any value of $\alpha$, then $S_{0}=-\infty$ for that value of $\alpha$, and $Q(\alpha)$ has a sharp peak at $G=0$. A randomly selected universe, then, is overwhelmingly likely to have $G=0$, in conflict with observation.

We must assume, therefore, that the bound eq. (4.1) applies. Then $Q(\alpha)$ favors that $G^{-1}$ assume a value that is close to its maximum in the region of $\alpha$-space where $A \sim R_{\max }^{-2}$; the typical deviation of $G^{-1}$ from its maximum is of order $R_{\max }^{-2}$. As the infrared cutoff is removed, $Q(\alpha)$ becomes a very sharply peaked function of both $\Lambda$ and $G$. The peak occurs precisely at the maximal value of $G^{-1}(\alpha)$ on the $\Lambda(\alpha)=0$ surface.

Presumably there are many $\alpha$ 's. The number of $\alpha_{a}$ 's is the number of nonzero eigenvalues of the matrix $\Delta_{a b}$ in eq. (2.1), and this number is likely to be infinite. 
The condition $\Lambda(\alpha)=0$ determines a surface of codimension one in the $\alpha$-space. But we should expect that the condition that $G(\alpha)$ assumes its minimum on the $\Lambda(\alpha)=0$ surface is sufficient to determine all of the remaining $\alpha$ 's. A function, even a function of many variables, generically attains its global minimum at an isolated point, assuming that a global minimum exists.

Therefore, we have in principle recovered the ability to calculate the constants of Nature. All of the arbitrariness that is potentially introduced by wormhole physics can be overcome. We need only compute all of the constants of Nature as functions of the $\alpha$ 's; then we find the $\alpha$ that minimizes $G$ on the $\Lambda=0$ surface. This determines the $\alpha$ 's, and hence all of the other constants. In practice, this calculation will be challenging.

The $\alpha$-dependence of $G$, or of any coupling constant, comes from two sources, and we can imagine carrying out the calculation of $G(\alpha)$ in two stages. First, we integrate out wormholes at the wormhole scale $M_{\mathrm{w}}$, and thus obtain a "bare" theory with cutoff $M_{\mathrm{w}}$ and $\alpha$-dependent "bare" parameters. Second, we allow the cutoff to float from $M_{\mathrm{w}}$ to $M \ll M_{\mathrm{w}}$; the effects of quantum fluctuations with wave number $k$ satisfying $M_{\mathrm{w}}>k>M$ are incorporated into a renormalization of the effective action. The fluctuations that are integrated out in the second stage are not wormholes, but conventional "loop" fluctuations. The $G(\alpha)$ we want to calculate is extracted from the effective action in the infrared or $M \rightarrow 0$ limit.

It is clear that if this $G(\alpha)$ is to have a nonvanishing minimum, then so must the bare Newton's constant $G_{0}(\alpha)$, renormalized at the scale $M_{\mathrm{w}}$. The point is that $G=0$ is stable under renormalization; Newton's constant is unrenormalized when gravity is free. (Renormalization in quantum gravity is discussed in more detail in sect. 6.) Therefore, if the bare Newton's constant $G_{0}(\alpha)$, obtained by integrating out wormholes, were to vanish for some $\alpha$, then the physical Newton's constant $G(\alpha)$ would also vanish for that value of $\alpha$. Since $G(\alpha)$ must satisfy the bound eq. (4.1), we require the bare coupling to satisfy a similar bound,

$$
G_{0}(\alpha) \geqslant G_{0, \min }>0
$$

In the dilute approximation described in sect. 2 , we saw that the effective action is linear in $\alpha$. Thus $G_{0}^{-1}(\alpha)$ is linear in $\alpha$, and $G_{0}$ vanishes when a particular combination of the $\alpha$ 's is infinite. In view of eq. (4.7), we conclude that the dilute approximation is invalid. To understand why the gravitational interaction has a nonvanishing strength we must go beyond the dilute approximation. The corrections to the dilute approximation are discussed in sect. 5, with particular attention devoted to the origin of the lower bound on $G_{0}(\alpha)$.

Is it possible that the requirement that $G(\alpha)$ attains its minimum on the $\Lambda(\alpha)=0$ surface fails to determine all the $\alpha$ 's? If there are undetermined $\alpha$ 's, then there is presumably a symmetry that prevents $G$ from depending on those $\alpha$ 's. But every- 
thing couples to gravity, and all other interactions contribute to the renormalization of $G$, so it is hard to think of such a symmetry.

We have emphasized in this section that Newton's constant $G(\alpha)$ must obey a positive lower bound; otherwise, on the basis of the same assumptions that underlie Coleman's explanation for $A=0$, we predict $G=0$, in conflict with observation. In fact, it is also necessary for the coefficients of higher derivative terms in the gravitational effective action to obey upper bounds. Just as the newtonian gravitational interaction must not be permitted to be arbitrarily weak for any value of $\alpha$, so the higher derivative gravitational interactions must not be permitted to be arbitrarily strong for any value of $\alpha$.

In the derivation of $A=0$ in sect. 3 we required that the contribution from higher derivative terms to the value of the effective action at the stationary point be negligible. A higher derivative term in the action with arbitrarily large coefficient can upset Coleman's mechanism by destabilizing the stationary point. Or the $\alpha$-distribution may favor that this coefficient be infinite; then the theory of gravity in a typical $\alpha$-universe will not agree with Newton's theory in the low-energy limit.

The parameters that must obey such upper bounds are coupling constants of the renormalized effective action that describes gravity at low energy. Unlike the lower bound on $G$, these upper bounds probably place no restrictions on the "bare" parameters of the effective theory defined at the wormhole scale $M_{\mathrm{w}}$. Rather, the upper bounds are an automatic consequence of the renormalization procedure as the cutoff floats from $M_{\mathrm{w}}$ to $M \ll M_{\mathrm{w}}$. All of the renormalized couplings of gravity obey upper bounds provided only that, as we expect, gravity has no nontrivial continuum limit ${ }^{\star}$. This will be explained further in sect. 6 .

\section{Beyond the dilute approximation}

Up to now in this paper we have for the most part treated wormholes as dilute, and have ignored the interactions among wormholes. But we have also noted that this dilute approximation cannot really be justified. In this section we will discuss the corrections to the dilute approximation due to interactions.

For the purpose of this discussion, we will find it useful to make a distinction between wormholes and "instantons." I will use the term instanton for the region of the background spacetime where a wormhole hooks on; the term instanton is appropriate because this is a small localized region in the background (euclidean) spacetime. A wormhole connects together two instantons.

We must distinguish two types of interactions that arise when corrections to the dilute approximation are considered; these may be called "wormhole interactions" and "instanton interactions." Wormhole interactions are described by vertices at

\footnotetext{
* A similar suggestion was made by Weinberg [14].
} 


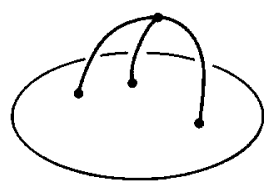

Fig. 10. A wormhole interaction.

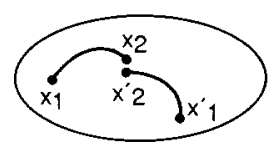

Fig. 11. A short-range interaction between instantons at spacetime positions $x_{2}$ and $x_{2}^{\prime}$.

which a branching of wormholes occurs (fig. 10). These interactions induce nongaussian corrections to the probability distribution $P(\alpha)$ in eq. (2.5) and eq. (3.2).

Of far greater interest are instanton interactions. These occur when two instantons closely approach one another on the background spacetime (fig. 11). The instanton interactions induce corrections to the $\alpha$-dependent effective action $S[g, \alpha]$ that are nonlinear in $\alpha$. Hence, the instanton interactions also correct the factor $Q(\alpha)$ in eq. (3.3). The instanton interactions are of greater interest than the wormhole interactions (at least for our present purposes) because, by modifying $Q(\alpha)$, they can change where the support of the $\alpha$-distribution lies.

If we assume that the instanton interactions are of short range, then the effects of these interactions can be systematically expanded in powers of a "density" of instantons. This expansion is precisely analogous to the cluster expansion of statistical mechanics [24]. The cluster expansion, when truncated at a finite order, gives accurate results if the range of the instanton interactions is small compared to the mean separation between instantons ${ }^{\star}$.

Actually, in a theory of gravity, we should not be surprised to find that instanton interactions are long-range. The instanton is likely to be surrounded by a gravitational field that decays like an inverse power of the distance from the instanton, and the gravitational interaction between instantons, then, will also decay like a power. (Indeed, there might be other light fields that are excited in the vicinity of the instanton, and these would also induce a long-range interaction.) The long-range interactions cause one to worry about the validity of the cluster expansion.

However, as Gupta and Wise [17] have recently emphasized, the long-range instanton interactions are not the effects that we are really interested in, because they do not generate corrections to the effective action $S[g, \alpha]$ that are nonlinear in $\alpha$. Instead, the physical effects of these long-range instanton interactions can be

\footnotetext{
^ The cluster expansion for QCD instantons was described in, for example, ref. [25].
} 
reproduced exactly in an effective field theory with an action linear in $\alpha$; in this effective theory, they are represented by exchanges of light field quanta between interaction vertices that are linear in $\alpha$. The terms in $S[g, \alpha]$ that are nonlinear in $\alpha$ are generated only by the genuinely short-range instanton interactions that occur when the separation between instantons is comparable to the instanton size. It is sensible to carry out an expansion of the effects of these interactions in powers of the instanton density. It will be understood, then, that the instanton interactions considered below are actually the short-range portion of interactions that may also include a long-range tail.

In order to formulate the cluster expansion, we must first understand how the notion of a density of instantons arises. For this purpose, we reconsider the dilute approximation that was described in sect. 2. In sect. 2 we made the hypothesis that the contribution $C$ of a single wormhole to a quantum mechanical expectation value can be expressed as a bilocal expansion in local operators, as in eq. (2.2). If the curvature of the background spacetime is small, and all fields on spacetime are weak (in units of the wormhole scale $R_{\mathrm{w}}^{-1}$ ), then the sum over local operators is dominated by the operator 1 , and the contribution becomes

$$
C=\frac{1}{2} \Delta V^{2}
$$

Here $V$ is the volume of the background spacetime, and $\Delta$ is the coefficient of the leading term in eq. (2.2), presumably of order $R_{\mathrm{w}}^{-8}$. In the semiclassical approximation discussed by Coleman [8] and Giddings and Strominger [9], $\Delta$ would be further suppressed by the small factor $\mathrm{e}^{-2 S}$, where $S$ is the (large) action of a semiwormhole.

In the dilute approximation, the contribution due to $N_{\mathrm{w}}$ noninteracting wormholes is $C^{N_{\mathrm{w}}} / N_{\mathrm{w}}$ ! The sum over $N_{\mathrm{w}}$ is thus dominated by $N_{\mathrm{w}}$ of order $C$. In other words, the number $N_{\mathrm{w}}$ of wormholes in a typical configuration that gives a significant contribution to the euclidean path integral is

$$
N_{\mathrm{w}}-\frac{1}{2} \Delta V^{2}
$$

It is therefore possible to regard $\Delta$ as a "density of wormholes" per volume squared. On the other hand, a configuration with $N$ wormholes has $2 N$ instantons; the notion of a "density of instantons" per volume does not appear to make sense in the $V \rightarrow \infty$ limit.

However, the above discussion applies to the combinatorics of wormholes only after the $\alpha$ integration in eq. (2.3) has been performed. (The effect of the integration is to match up $2 N_{\mathrm{w}}$ instantons with $N_{\mathrm{w}}$ wormholes in all possible ways.) Instead, we are interested in studying physics at a fixed value of $\alpha$. The leading factor in the integrand of eq. (2.3), arising from the operator 1 in the expansion, has the form

$$
\mathrm{e}^{(1 / 2) \alpha^{2}} \mathrm{e}^{\alpha \Delta^{1 / 2 \nu}}
$$


(Here we have rescaled $\alpha$ so that the wormhole "propagator" is one; $\alpha$ is now dimensionless.) The term in eq. (5.3) of order $V^{N}$ may be interpreted as the contribution at fixed $\alpha$ due to an $N$-instanton configuration, and the sum over $N$ is dominated by

$$
N / V \sim \alpha \Delta^{1 / 2} .
$$

Thus, $\alpha \Delta^{1 / 2}$ may be regarded as a density of instantons.

There is a peculiar complementarity at work here. We began this discussion by describing the physical effects of bilocal objects, the wormholes. But then, for sound physics reasons, we focused attention on a sector with a fixed value of $\alpha$. For $\alpha$ fixed, it is far more appropriate to describe the physics in terms of local objects, the instantons, instead of in terms of wormholes. Furthermore, while the density of wormholes is, of course, $\alpha$-independent, the density of instantons depends on $\alpha$ as in eq. (5.4).

The instanton density $\alpha \Delta^{1 / 2}$ will be the expansion parameter of our cluster expansion, and the dilute approximation is a good approximation of $\alpha \Delta^{1 / 2}$ sufficiently small. Thus, whatever the value of $\Delta^{1 / 2}$, the dilute approximation can be justified for small enough $\alpha$. (And this in no way requires the validity of a semiclassical approximation; that is, wormhole effects need not be dominated by the contribution of a classical wormhole solution.) On the other hand, whatever the value of $\Delta^{1 / 2}$, the dilute approximation will always break down for $\alpha$ sufficiently large. In particular, the dilute approximation cannot be used to study the behavior of the distribution $Q(\alpha)$ for asymptotically large $\alpha$.

In the dilute approximation, it is assumed that instantons can be freely superposed, and that a contribution due to two instantons factorizes into a product of one instanton contributions. But this assumption is expected to fail when the instantons closely approach one another. More generally, the two-wormhole configuration depicted in fig. 11, in which instantons centered at $x_{2}$ and $x_{2}^{\prime}$ have a small separation, gives a contribution

$$
\begin{aligned}
& \frac{1}{8} \int \mathrm{d}^{4} x_{1} \sqrt{g_{1}} \mathrm{~d}^{4} x_{2} \sqrt{g_{2}} \mathrm{~d}^{4} x_{1}^{\prime} \sqrt{g_{1}^{\prime}} \mathrm{d}^{4} x_{2}^{\prime} \sqrt{g_{2}^{\prime}} \\
& \quad \times \sum_{a, b, a^{\prime}, b^{\prime}} \mathcal{O}_{a}\left(x_{1}\right) \Delta_{a b}\left[\mathcal{O}_{b}\left(x_{2}\right) \mathcal{O}_{b^{\prime}}\left(x_{2}^{\prime}\right)+R_{b b^{\prime}}^{(2)}\left(x_{2}, x_{2}^{\prime}\right)\right] \Delta_{b^{\prime} a^{\prime}} \mathcal{O}_{a^{\prime}}\left(x_{1}^{\prime}\right)+\ldots
\end{aligned}
$$

Here, the remainder $R_{b b^{\prime}}^{(2)}\left(x, x^{\prime}\right)$ is a small correction to the factorized operator $\mathcal{O}_{b}(x) \mathcal{O}_{b}\left(x^{\prime}\right)$ when $\left(x-x^{\prime}\right)$ is large compared to the characteristic instanton size. (Of course, there are also corrections that occur when other pairs of instantons closely approach one another; these have not been indicated in eq. (5.5).)

If we include the effects of all two-instanton interactions in the wormhole sum, the integrand of the $\alpha$-integration in eq. (2.3) becomes modified. The modification is 
a shift in the effective action of order $\alpha^{2}$,

$$
\begin{aligned}
\delta S^{(2)}(\alpha) & =-\frac{1}{2} \sum_{a, b} \alpha_{a} \lambda_{a b}^{(2)} \alpha_{b} \\
\lambda_{a b}^{(2)} & =\int \mathrm{d}^{4} x \sqrt{g} \mathrm{~d}^{4} x^{\prime} \sqrt{g^{\prime}} R_{a b}^{(2)}\left(x, x^{\prime}\right) .
\end{aligned}
$$

It is clear that the "Feynman rule" for evaluating the $\alpha$-integral tells us that the effect of $\delta S^{(2)}(\alpha)$ is to include the two-instanton interaction for all pairs of instantons.

Similarly, there are (connected) $n$-body instanton interactions that occur when $n$ instantons closely approach one another. Their effect is to induce an order- $\alpha^{n}$ shift in the effective action

$$
\begin{aligned}
\delta S^{(n)}(\alpha) & =-\frac{1}{n !} \sum_{a_{1} \ldots a_{n}} \alpha_{a_{1}} \ldots \alpha_{a_{n}} \lambda_{a_{1} \ldots a_{n}}^{(n)}, \\
\lambda_{a_{1} \ldots a_{n}}^{(n)} & =\int \mathrm{d}^{4} x_{1} \sqrt{g_{1}} \ldots \mathrm{d}^{4} x_{n} \sqrt{g_{n}} R_{a_{1} \ldots a_{n}}^{(n)}\left(x_{1}, \ldots, x_{n}\right),
\end{aligned}
$$

where $R_{a_{1} \ldots a_{n}}^{(n)}$ is a translation-invariant operator-valued function that drops off rapidly when any separation $\left(x_{i}-x_{j}\right)$ is large.

If the instanton interactions are of short range, then the interaction vertex $R^{(n)}$ in eq. (5.7) is smeared out in spacetime over a region with a size comparable to the size of an instanton. The effects of this interaction at low energy can be well accounted for by a local effective action. Formally, we may perform $n-1$ of the integrals over spacetime in eq. (5.7); we then obtain one remaining integral over spacetime of a function that can be expanded in terms of local operators.

By thus expanding eq. (5.7) in terms of local operators, we may express

$$
S(\alpha)=S^{(0)}+\sum_{n=1}^{\infty} S^{(n)}(\alpha)
$$

as a sum of local operators, with the coefficient of each operator expanded as a power series in $\alpha$. For example, the coefficient $\tilde{\Lambda}_{0}$ of $\int \mathrm{d}^{4} x \sqrt{g}$ may be written ${ }^{\star}$

$$
\tilde{\Lambda}_{0}(\alpha)=\tilde{\Lambda}^{(0)}+\sum_{n=1}^{\infty} \sum_{a_{1} \ldots a_{n}} \frac{1}{n !} \tilde{\Lambda}_{a_{1} \ldots \ldots a_{n}}^{(n)} \alpha_{a_{1}} \ldots \alpha_{a_{n}} .
$$

* $\tilde{\Lambda}_{0}$ is $\Lambda_{0} / 8 \pi G_{0}$ in the notation of eq. (3.5). It is a "bare" parameter that will be further renormalized by fluctuations below the wormhole scale $M_{\mathrm{w}}$. 
Expansions like eq. (5.9) are the basis of our earlier claim that corrections due to instanton interactions may be expanded in powers of the instanton density. We now see that this statement is true only rather schematically. The term $\Sigma_{a} \Lambda_{a}^{(1)} \alpha_{a}$ linear in $\alpha$ in eq. (5.9) is the density of instantons that we identified in eq. (5.4). The terms higher order in $\alpha$ are not really higher powers of the density; each term involves a different combination of the $\alpha^{\prime} \mathrm{s}^{\star}$. But, generically, when the mean instanton separation is comparable to the instanton size, all terms in eq. (5.9) are of roughly the same order.

Borrowing metaphorically from the semiclassical picture, we might regard each $\alpha_{a}$ as being associated with a distinct instanton "type." Then each $\alpha_{a}$ can be interpreted as the density of instantons of type $a$, and eq. (5.9) is a power series expansion in the various densities.

There is an expansion similar to eq. (5.9) for $G_{0}^{-1}$, where $G_{0}$ is the bare Newton's constant at the wormhole scale $M_{\mathrm{w}}$. We noted in sect. 4 that, if we are to accept Coleman's explanation for the vanishing of the cosmological constant, we must insist that $G_{0}^{-1}$ is bounded above. Obviously, we cannot understand the origin of this bound by considering the expansion in $\alpha$ to any finite order. We must somehow sum up the series.

Before discussing further the upper bound on $G_{0}^{-1}$, let us notice that there is another logically independent reason why we must go beyond the dilute approximation if we are to invoke Coleman's mechanism. If we compute quantum corrections in the effective theory cut off at the wormhole scale $M_{\mathrm{w}}, \tilde{\Lambda}$ will be renormalized by an amount of order $M_{\mathrm{w}}^{4}$. It would presumably require an unnatural fine tuning for the $\alpha$-independent bare parameter $\tilde{\Lambda}^{(0)}$ to cancel this renormalization to high accuracy. Therefore, if the renormalized cosmological constant is to vanish, the $\alpha$-dependent shift of $\tilde{\Lambda}_{0}(\alpha)$ in eq. (5.9) must be at least of order $M_{\mathrm{w}}^{4}$. But then, the density of instantons is of order $M_{\mathrm{w}}^{4}$, or the mean instanton separation is $M_{\mathrm{w}}^{-1}=R_{\mathrm{w}}$, which is just the condition for corrections to the dilute approximation to be important ${ }^{\star \star}$.

We cannot escape this conclusion by appealing to supersymmetry. If supersymmetry remains unbroken below the wormhole scale, then $\tilde{\Lambda}_{0}(\alpha)=0$. But a bare cosmological constant is eventually generated at a lower scale, where supersymmetry is spontaneously broken. Again, unless the $\alpha$-independent bare parameters are carefully tuned, we expect that a large $\alpha$-dependent shift of bare parameters is required for the renormalized cosmological constant to vanish. The $\alpha$-dependent shifts are small when the instantons are dilute.

Let us now consider further the origin of the bound on Newton's constant. Here we wish to argue that it is plausible that $G_{0}^{-1}(\alpha)$, the bare coupling at the wormhole

\footnotetext{
* Indeed, for the other coefficients in $S(\alpha)$, even the linear term involves a different linear combination of the $\alpha$ 's than appears in the "instanton density."

$\star \star$ This argument emerged from discussions with A. Cohen and M. Wise.
} 
scale $M_{\mathrm{w}}$, is bounded above as a function of $\alpha$. The renormalization of $G^{-1}$ by quantum fluctuations below the wormhole scale will be discussed in sect. 6 .

Heuristically, one expects instanton effects to "saturate" when the mean instanton separation becomes comparable to the instanton size $R_{\mathrm{w}}$. When the instantons become dense, the picture on which our analysis has been based - of wormholes attaching to a smooth background spacetime - may no longer apply. Indeed, the notion of an instanton gas, and the interpretation of our expansion parameter as a density of instantons, probably ceases to make sense when the dilute approximation is no longer valid.

It is reasonable to guess that, if the $\alpha$-dependent shift of a parameter in the effective action does have a maximum as a function of $\alpha$, then the maximum is attained at a value of $\alpha$ that is comparable to the value of $\alpha$ for which the cluster expansion breaks down. That is, the maximum occurs when the instanton density is of order one. We would estimate then, that the maximal shift is a factor of order one times a power of the wormhole scale $M_{\mathrm{w}}$ determined by dimensional analysis. For example, for Newton's constant we might expect

$$
\delta\left[\left(16 \pi G_{0}\right)^{-1}\right] \lesssim M_{\mathrm{w}}^{2}
$$

to be satisfied for any $\alpha$.

To make this discussion a bit more concrete, I will describe a simple toy model in which the cluster expansion can be summed to all orders in $\alpha$. In this toy model, we can see explicitly that $G_{0}^{-1}$ is a bounded function of $\alpha$. For the sake of simplicity, this model will have just one "type" of instanton and just one $\alpha$-parameter; it is obviously possible to generalize it to a model with many $\alpha$ 's and with qualitatively similar behavior. The point of the model is that excluded volume effects can be expected to cause repulsive interactions of instantons at small separations, and that short-range repulsive interactions can significantly alter the $\alpha$-dependence of the effective action at asymptotically large $\alpha$.

For the purpose of characterizing the short-range instanton interactions in this toy model, imagine that euclidean spacetime has been divided into many identical tiny cells, each with a physical volume of $R_{\mathrm{w}}^{4}$, the volume of an instanton. A configuration of the instanton gas can be described by assigning to each cell a non-negative integer, the number of instantons that occupy the cell (see fig. 12). We will label the cells with an index $i$, and denote the corresponding integer by $n_{i}$.

Now the assumption of our model is that instantons in distinct cells do not interact, but instantons in the same cell $d o$ interact. An instanton occupying cell $i$ may either encourage more instantons to occupy that cell (attractive interaction), or discourage additional instantons (repulsive interaction).

Suppose, at first, that there are no instanton interactions at all. Then the instantons are noninteracting identical particles, and summing over all configura- 


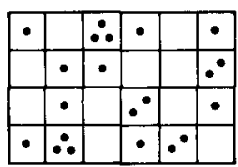

Fig. 12. Euclidean spacetime has been divided into cells; each cell contains a number of instantons that is assumed to be a (non-negative) integer.

tions of the instanton gas generates an effective action $\delta S(\alpha)$ given by

$$
\begin{aligned}
\mathrm{e}^{-\delta S(\alpha)} & =\prod_{i}\left(\sum_{n_{i}=0}^{\infty} \frac{1}{n_{i} !}\left(\alpha \mathcal{O}_{i}\right)^{n_{i}}\right)=\prod_{i} \mathrm{e}^{\alpha \mathcal{O}_{i}} \\
& =\exp \left(\alpha \sum_{i} \mathcal{O}_{i}\right) .
\end{aligned}
$$

Here $\mathcal{O}_{i}$ quantifies the dependence of the instanton contribution on the background geometry; it can be expanded in terms of local operators in the cell $i$. In eq. (5.11) we have recovered the result eq. (2.4) that we derived previously in the dilute approximation.

To simulate interactions, we replace the sum over $n$ in eq. (5.11) by

$$
\sum_{n=0}^{\infty} \frac{a_{n}}{n !}\left(\alpha O_{i}\right)^{n}
$$

Then, for $a_{n}>1$, we have introduced an attractive short-range $n$-body interaction, and for $a_{n}<1$, a repulsive $n$-body interaction. With this modification, eq. (5.11) becomes

$$
\begin{aligned}
\delta S(\alpha) & =-\sum_{i} \ln f\left(\alpha \mathcal{O}_{i}\right), \\
f(z) & =\sum_{n} \frac{a_{n}}{n !} z^{n} .
\end{aligned}
$$

To extract the value of $\delta G_{0}^{-1}$, we expand $\delta S$ in powers of derivatives of the background metric. If the operator $\mathcal{O}$ can be expanded as

$$
\mathcal{O}=c_{0}+c_{1} R+\ldots
$$

(where $R$ is the Ricci scalar), then we have

$$
\delta S(\alpha)=\int \mathrm{d}^{4} x \sqrt{g} R_{\mathrm{w}}^{-4}\left(-\ln f(\tilde{\alpha})-\frac{\tilde{\alpha} f^{\prime}(\tilde{\alpha})}{f(\tilde{\alpha})} \frac{c_{1}}{c_{0}} R(x)+\ldots\right),
$$


where

$$
\tilde{\alpha}=c_{0} \alpha
$$

In eq. (5.14), the sum over cells has been replaced by a volume integral, and the volume $R_{\mathrm{w}}^{4}$ of the cell has been inserted.

From eq. (5.14) we see that the shift in Newton's constant is given by

$$
\delta\left[\left(16 \pi G_{0}\right)^{-1}\right]=\frac{\tilde{\alpha} f^{\prime}(\tilde{\alpha})}{f(\tilde{\alpha})} \frac{c_{1}}{c_{0} R_{\mathrm{w}}^{4}} .
$$

There are many reasonable functions $f(\tilde{\alpha})$ for which this shift is bounded; for example, $f$ may be an (even) real polynomial with no real zeros. A simple function that works is

$$
f(z)=1+z+\frac{1}{2} z^{2}
$$

In this case, one or two instantons are allowed to occupy a cell without interacting, but a repulsive interaction forbids three or more instantons in a single cell.

Clearly this toy model has many unrealistic features, but it serves to illustrate that instanton interactions can cause $\alpha$-dependent shifts in the effective action to be bounded as functions of $\alpha$.

Before concluding this section, I would like to reexamine one of the assumptions underlying our whole analysis, in the light of our observations about instanton interactions. This assumption was explicitly stated in sect. 2 - that wormholes have a characteristic thickness $R_{\mathrm{w}}$. Then it makes sense to integrate out wormholes, and obtain an effective theory with cutoff $R_{\mathrm{w}}^{-1}$, in which wormhole fluctuations no longer occur. The heuristic picture underlying this assumption is that thick wormholes have large euclidean action, and so give a contribution to the path integral that is highly suppressed. We therefore make only a very small error by neglecting wormholes that are much thicker than the characteristic size $R_{\mathrm{w}}$.

This reasoning, however, must be regarded with caution. We may imagine that the $\alpha$ 's can be divided into two sets - parameters $\alpha_{\mathrm{S}}$ associated with small instantons and parameters $\alpha_{L}$ associated with large instantons. On the basis of the heuristic picture described above, we expect that the "propagator" $\Delta_{L}$ for large wormholes is extremely small; the probability distribution $P(\alpha)$ in eq. (2.5) strongly favors small values of $\alpha_{\mathrm{L}}$. It is in this sense that large $\alpha_{\mathrm{L}}$-dependent shifts in the effective action are highly improbable.

But as we emphasized in sect. 3 , it is not $P(\alpha)$ but the factor

$$
Q(\alpha) \sim \exp \{\exp [3 \pi / G(\alpha) \Lambda(\alpha)]\}
$$

that determines where the support of the $\alpha$-distribution lies. The very singular 


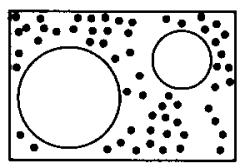

Fig. 13. Large instantons exclude small instantons from spacetime.

dependence of eq. (5.18) on $\alpha_{\mathrm{L}}$ for $\Lambda(\alpha) \sim 0$ overcomes the $\exp \left(-\alpha_{\mathrm{L}}^{2} \Delta_{\mathrm{L}}\right)$ suppression in $P(\alpha)$. Hence, in spite of their large euclidean action, the large instantons try their best to increase $G^{-1}$ on the $A=0$ surface. The contribution due to large instantons to $G^{-1}$ is presumably maximized when the large instantons are dense. And so, in spite of the strong tendency of the factor $P(\alpha)$ to suppress the effects of the large instantons, the large instantons cannot be prevented from becoming dense, and inducing effects that are of order one.

This conclusion is distressing. It appears that wormholes of arbitrarily large thickness will contribute significantly to physical processes at low energy. How are we to reconcile this phenomenon with the well-documented success of local field theory in describing low-energy physics ${ }^{\star}$ ?

I believe that interactions between large and small instantons are responsible for suppressing the effects of large instantons. I have in mind a picture in which small instantons crowd out the large ones (fig. 13). If our goal is to maximize $G^{-1}$, then configurations with many large instantons are inefficient, because the large instantons exclude from spacetime a region that the small instantons would like to occupy. A dense gas of small instantons gives a contribution of order $R_{\mathrm{S}}^{-2}$ to $G_{0}^{-1}$, where $R_{\mathrm{S}}$ is the size of a small instanton, while a dense gas of large instantons contributes of order $R_{\mathrm{L}}^{-2}$, where $R_{\mathrm{L}}$ is the size of a large instanton. Therefore, if there is a trade-off between large and small instantons, the instanton gas will favor increasing the abundance of small instantons at the expense of the large instantons. This effect might provide the justification for ignoring the large wormholes.

A very crude model will illustrate how this mechanism works. Imagine that there are only two instanton types, the small ones with size $R_{\mathrm{S}}$ and the large ones with size $R_{\mathrm{L}}$. Then the $\alpha$-dependence of the bare Newton's constant has the approximate form

$$
G_{0}^{-1} \cong C_{\mathrm{S}} R_{\mathrm{S}}^{-2} n_{\mathrm{S}}\left(\alpha_{\mathrm{S}}\right)+C_{\mathrm{L}} R_{\mathrm{L}}^{-2} n_{\mathrm{L}}\left(\alpha_{\mathrm{L}}\right)
$$

Here $n_{\mathrm{S}}$ is the dimensionless density of small instantons, the fraction of the volume of spacetime that is occupied by the instantons; $n_{\mathrm{L}}$ is the corresponding density of large instantons, and $C_{\mathrm{S}}, C_{\mathrm{L}}$ are numerical constants of order one. We wish to determine $\alpha_{\mathrm{S}}$ and $\alpha_{\mathrm{L}}$ by maximizing $G_{0}^{-1}$. But if small instantons cannot sit on top

\footnotetext{
* This issue was also raised by Kaplanovsky [20], and has been discussed in refs. [16,18].
} 


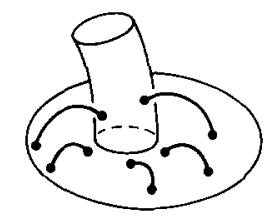

Fig. 14. If excluded from attaching to spacetime by a large instanton, a small wormhole may attach to a large wormhole instead.

of large instantons, then the densities are constrained by

$$
n_{\mathrm{S}}\left(\alpha_{\mathrm{S}}\right)+n_{\mathrm{L}}\left(\alpha_{\mathrm{L}}\right) \leqslant 1
$$

Evidently, the maximum of $G_{0}^{-1}$ subject to the constraint eq. (5.20) is $n_{\mathrm{S}}=1$, $n_{\mathrm{L}}=0$, assuming that $C_{\mathrm{S}} R_{\mathrm{S}}^{-2}>C_{\mathrm{L}} R_{\mathrm{L}}^{-2}$. The large instantons are completely eliminated in favor of the small instantons.

The distinction between instanton interactions and wormhole interactions is important for understanding why large and small instantons mutually exclude each other. When excluded from spacetime by a large instanton, a small instanton will slide up onto a large wormhole (fig. 14); that is, the small wormhole will attach to the large wormhole instead of attaching to the background spacetime. One might have argued from this perspective that the physics of small wormholes is unaffected by large wormholes; the small wormhole finds the large wormhole indistinguishable from the background spacetime. But we have already learned that the physical consequences of wormhole interactions are quite different than the consequences of instanton interactions. The interactions between large and small wormholes alter the distribution $P(\alpha)$, but have no effect on the crucial factor $Q(\alpha)$ that determines what values of the $\alpha$ 's are favored. The instanton interactions, not the wormhole interactions, affect $Q(\alpha)$, and it is therefore the instanton interactions, not the wormhole interactions, that must account for the suppression of the effects of large wormholes.

The suggestion that small instantons are responsible for suppressing the effects of large instantons sounds surprising at first. It is a cherished principle of physics, the decoupling principle, that long-distance physics is relatively insensitive to the details of short-distance physics. Ordinarily, we expect that it is possible to "integrate out" short-wavelength quantum fluctuations and incorporate the effects of these fluctuations into the renormalization of the parameters of an effective field theory that describes long-distance physics. (Indeed, we invoked this strategy in sect. 3, and will do so again in sect. 6.) Hence, we might expect that the effects of large instantons can be understood within the context of a low-energy effective field theory in which small instantons have already been integrated out. But how can the mechanism 
described above for suppressing large instantons, which arises from interactions with small instantons, be understood in such an effective field theory?

I believe, in fact, that the $\alpha$-dependence of $G^{-1}$ is an exception to the decoupling principle. The dominant contribution to the dependence of $G^{-1}$ on $\alpha_{\mathrm{L}}$ cannot be accounted for in terms of long-distance physics alone. It arises because of the unusual way in which short-distance physics and long-distance physics are intertwined here. Usually, we may think of short-wavelength fluctuations as being superposed on a long-wavelength background field configuration. Because the short-wavelength fluctuations are indifferent to the long-wavelength physics, they induce renormalizations that are independent of the background field; that is why decoupling usually works. From this perspective, the key feature of the instanton interactions is that they prevent small instantons from sitting on top of large instantons. Therefore, the effects of the small instantons are not independent of the configuration of large instantons. The dependence of $G^{-1}$ on $\alpha_{\mathrm{L}}$ is thus dominated by the effect of $\alpha_{\mathrm{L}}$ on the small instantons, and it cannot be understood in terms of long-distance physics alone. That is why decoupling fails.

One should also notice that this mechanism for suppressing large instantons relies crucially on the claim that the $\alpha$ 's are determined by maximizing $G^{-1}$. The point is simply that $G^{-1}$ has the dimensions of mass to a positive power. Therefore, merely on dimensional grounds, small instantons are much more effective than large instantons at causing $G^{-1}$ to increase.

\section{Renormalization in quantum gravity}

In spite of the intrinsic indeterminacy introduced by wormhole effects, it is reasonable to hope that the constants of Nature can, in principle, be computed. We must calculate the dependence on the $\alpha$ 's of the various parameters that characterize low-energy physics, and then find the value of $\alpha$ that is overwhelmingly favored by the probability distribution $Q(\alpha)$ in eq. (3.3).

To find the favored value of $\alpha$, it is enough to know the $\alpha$-dependence of two quantities, the (renormalized) cosmological constant $\Lambda$ and the (renormalized) Newton's constant $G$. As we have emphasized, the $\alpha$-dependence of these quantities arises from two sources (as does the $\alpha$-dependence of all the renormalized parameters), and we can imagine calculating the $\alpha$-dependence in two stages. In the first stage, we integrate out wormhole fluctuations, and thus obtain an effective theory with $\alpha$-dependent bare couplings that is cut off at the wormhole scale $M_{\mathrm{w}}$. In the second stage, we integrate out quantum fluctuations with wavelengths greater than $R_{\mathrm{w}}=M_{\mathrm{w}}^{-1}$, to obtain an effective theory that is appropriate for describing the far-infrared behavior of gravity. The first stage was the subject of sect. 5 , and the second stage is the subject of this section.

In sect. 5, we argued that it will be difficult to analyze the first stage with precision, because the dilute approximation does not apply. This conclusion already 
discourages us about the prospects for calculating the $\alpha$-dependence of $\Lambda$ and $G$. But even if it is hopeless to calculate the $\alpha$-dependence of the bare couplings induced at the first stage, one might still nurture the hope that the preferred values of at least some of the renormalized couplings can be calculated to reasonable accuracy. There are, presumably, many $\alpha$ 's, and so it may not be seriously wrong to assume that, as the $\alpha$ 's vary, the bare couplings at the wormhole scale can vary without restriction ${ }^{\star}$. Then we can forget all about the origin of the $\alpha$-dependence. Our task is simply to find "the best of possible worlds" - that choice of the bare parameters at scale $M_{\mathrm{w}}$ for which Coleman's distribution has its sharp peak.

Unfortunately, this program in its most extreme form suggested above is doomed to fail. As we have seen, in the best of worlds $G$ vanishes as well as $\Lambda$, and there is no gravitational interaction. We must accept that our universe is not the most perfect one, and abandon the hypothesis that wormhole effects allow the bare parameters to vary arbitrarily. A milder version of this hypothesis might be reasonable, however; we can assume that while $G_{0}^{-1}$ is bounded above as a function of the $\alpha$ 's, all other bare couplings can be varied without restriction. Surely, this is at best a caricature of the actual $\alpha$-dependence of the bare couplings, but it may serve as a first approximation.

This point of view suggests some questions that we will address below. For example, will an upper bound on $G_{0}^{-1}$ suffice to ensure that the renormalized $G^{-1}$ is also bounded above, as we require for Coleman's mechanism to be consistent with observation, even if no restrictions are placed on other bare couplings? And also, do the coefficients in the renormalized gravitational effective action of terms that involve higher derivatives of the metric obey suitable bounds even as the bare couplings vary without restriction? Such bounds are also required for the consistency of Coleman's mechanism.

I will argue that Coleman's explanation for $\Lambda=0$ passes both of the consistency tests posed above. That is, only one condition on the $\alpha$-dependence of the base couplings - the upper bound on $G_{0}^{-1}$ - suffices to justify Coleman's picture of the infrared behavior of gravity. These arguments are rather heuristic, but, I hope, cogent.

What can be said about the calculability, in practice, of the constants of Nature (other than the cosmological constant)? With respect to this issue, there is not much cause for encouragement. We hope to calculate the various renormalized parameters that describe low-energy physics. For this purpose, we must find the dependence of the renormalized $G$ on these renormalized quantities. This dependence might have been calculable if the renormalization of $G$ were dominated by long-wavelength effects that could be naturally expressed in terms of couplings renormalized at low energy. But unfortunately, the renormalization of $G$ is actually dominated by

* This means, of course, that the bare action varies "without restriction" within a space of reasonable quasilocal theories. 
short-wavelength quantum fluctuations. Therefore, the preferred values of the constants of Nature are actually sensitive to physics at the wormhole scale $M_{\mathrm{w}}$ and cannot be computed based on low-energy physics alone.

In a more optimistic light, by failing to make predictions beyond $\Lambda=0$ we have avoided making any wrong predictions that would call Coleman's mechanism into question. Indeed, based on a different point of view than advocated here, Grinstein and Wise [15] and Klebanov, Susskind, and Banks [16] reached some unfortunate conclusions - for example, that the pion has vanishing mass. This embarrassment is evaded if, as I claim, the preferred values of the renormalized quantities are actually determined by short-distance physics. Of course, it remains to be seen whether "the best of possible worlds" in the revised sense proposed here will resemble the universe that we observe.

Let us now consider in more detail the relation between the bare theory at the wormhole scale $M_{\mathrm{w}}$ and the renormalized theory that describes physics at very low energy. It is convenient to imagine that the renormalized theory is obtained from the bare theory by means of a renormalization group transformation [23]. The bare theory is cut off at the mass scale $M_{\mathrm{w}}$ and is quasilocal on the distance scale $M_{\mathrm{w}}^{-1}$. This bare theory might be quite complicated, involving many degrees of freedom and interactions. Now we allow the cutoff to float down from $M_{\mathrm{w}}$ to $M \ll M_{\mathrm{w}}$; we integrate out all quantum fluctuations with wave number between $M_{\mathrm{w}}$ and $M$, and we incorporate the effects of these fluctuations into renormalized parameters of an effective theory that has cutoff $M$ and is quasilocal on the distance scale $M^{-1}$. After $M$ has descended below the mass of the lightest massive particle in the original theory, the only remaining degrees of freedom in the effective theory are massless fields, such as the graviton and photon. It is the parameters of this effective theory, in the limit $M \rightarrow 0$, that enter into Coleman's calculation of the distribution $Q(\alpha)$.

The $M \rightarrow 0$ limit of the effective theory is the "continuum limit" of the bare theory that we started with. It describes the physics of the theory on length scales that are arbitrarily large compared to the original short-distance cutoff. Furthermore, the only surviving interaction in the $M \rightarrow 0$ limit is gravity.

Other massless particles (such as the photon) may remain coupled to the graviton, but the self-interactions of matter have been integrated out. (For example, there is no electrodynamic interaction because there are no massless charged particles.) Thus, the $M \rightarrow 0$ limit is the continuum limit of a cutoff theory of gravity. In fact, as we allow $M$ to float toward zero, the renormalized cosmological constant $\Lambda$ must also approach zero, as we discussed in sect. 3 . So we are considering the continuum limit of gravity with a vanishing cosmological constant.

This is a useful insight, because Einstein gravity is nonrenormalizable in perturbation theory, and it would be a stunning surprise if it turned out to be possible to take a continuum limit of quantum gravity other than free field theory. In other words, if the strength of gravitational effects is held fixed at the mass scale $M$, we do not expect to be able to push the cutoff mass $M_{0}$ up arbitrarily high, while 
maintaining a quasilocal theory of gravity at the scale $M_{0}$. If this were possible, then we could devise an ordinary field theory of gravity that makes sense at arbitrarily short distances, just as quantum chromodynamics (presumably) can describe the strong interaction at arbitrarily short distances. Then we would have a consistent field theory of quantum gravity; there would be no need for strings.

The conjecture that quantum gravity has no nontrivial continuum limit can be stated from two different points of view. We may consider (as above) physics at scale $M$ to be fixed, and then ask whether a bare theory can be constructed that reproduces the physics at scale $M$ as the cutoff $M_{0}$ of the bare theory approaches infinity. Alternatively, we may consider the cutoff $M_{0}$ of the bare theory to be fixed, and then ask whether a gravitational interaction at scale $M$ can remain nontrivial as $M \rightarrow 0$, for any choice of the quasilocal bare theory with cutoff $M_{0}$.

The effective theory at scale $M$ has an action $S_{M}$ that can be expanded in terms of (quasi-)local operators. Each operator has a coefficient that can be made dimensionless by multiplying by an appropriate power of $M$. Thus, $S_{M}$ can be specified by an infinite set of dimensionless couplings ${ }^{\star}, \lambda_{M}^{a}, a=1,2,3, \ldots$. The conjecture that gravity has no continuum limit, then, in the second form mentioned above, can be stated: For any choice of the quasilocal bare theory at the cutoff $M_{0}$, all dimensionless renormalized couplings $\lambda_{M}^{a}$ approach zero as $M \rightarrow 0^{\star \star}$. In fact, one expects that for small nonvanishing $M$, the $\lambda_{M}^{a}$ 's obey a somewhat stronger inequality constraint,

$$
\lambda_{M}^{a} \leqslant \lambda_{\max }^{a}\left(\frac{M_{0}}{M}\right), \quad M \ll M_{0},
$$

where the upper bound $\lambda_{\max }^{a}$, a dimensionless function of $M_{0} / M$, approaches zero for $M_{0} / M \rightarrow \infty$.

The inequality eq. (6.1) holds for any choice of the quasilocal bare theory at cutoff scale $M_{0}$, even as the bare couplings range without bound; there is no renormalized theory at scale $M$ that is descended from a quasilocal bare theory at scale $M_{0}$ and that has $\lambda_{M}^{a}$ greater than $\lambda_{\text {max }}^{a}\left(M_{0} / M\right)$. One might have supposed that we can specify any renormalized theory we please at scale $M$ and then obtain the corresponding bare theory at scale $M_{0}$ by running the renormalization group transformation in reverse. But this typically fails, because the renormalization group flow reaches a boundary of the space of quasilocal theories before the floating cutoff $M_{0}$ is attained.

The claim that the renormalized couplings are bounded even though the bare couplings are not, may sound surprising at first, but this behavior is just the

\footnotetext{
"Strictly speaking, these are the "essential" couplings that are not changed by local redefinitions of the fields $[23,26]$.

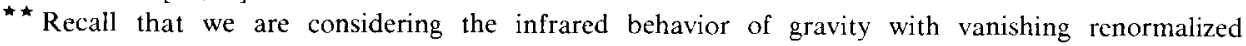
cosmological constant.
} 
(nonperturbative) "Landau ghost" phenomenon. The Landau ghost is familiar in, for example, QED and $\phi^{4}$ theory in four dimensions, two field theories that, though perturbatively renormalizable, are not expected to have nontrivial continuum limits $[23,27]$. It is characteristic of this phenomenon that a renormalized coupling $\lambda_{M}$, when evolved by means of the renormalization group to a larger renormalization scale, attains an infinite value at a finite scale $M^{\prime}>M$. Equivalently, Landau ghost behavior means that renormalized couplings at scale $M$ obey the inequality eq. (6.1), even as bare couplings at the cutoff $M_{0}$ vary without restriction. Physically, very strong charge screening effects keep the renormalized coupling finite even if the bare coupling is infinite.

We conclude, then, that if no quantum field theory of gravity has a continuum limit, then all gravitational effects must become weak in the $M \rightarrow 0$ limit, irrespective of the bare theory defined at scale $M_{0} \gg M$; all dimensionless couplings $\lambda_{M}^{a}$ approach zero. Because all dimensionless couplings are small, the renormalization group flow can be reliably computed for $M \ll M_{0}$ in perturbation theory. This perturbative analysis allows us to justify neglecting terms in the effective action that involve higher derivatives of the metric in the derivation in sect. 3. (The perturbative flow equations will be discussed further below.) Thus, Coleman's explanation for $\Lambda=0$ has passed a nontrivial consistency test; we need not worry about higherderivative interactions upsetting his mechanism, regardless of the $\alpha$-dependence of the bare higher-derivative couplings.

Like the other dimensionless couplings, $G_{M} M^{2}$ obeys the inequality eq. (6.1). Perturbative power-counting as described below then shows that $G_{M}$ stops running as $M \rightarrow 0$; it approaches the limit $G$, the renormalized Newton's constant. Therefore $G$ obeys an inequality

$$
G<C M_{0}^{-2},
$$

where $C$ is a constant, presumably of order one, independent of the bare theory at the cutoff $M_{0}$. Correspondingly, the coefficient $G^{-1}$ of $R$ in the renormalized effective action eq. (3.5) is bounded from below. In particular, then, $G^{-1}$ is positive, as we assumed in sect. 3 .

But there is no lower bound on $G$ that we can infer from a renormalization group argument alone. Indeed, all gravitational interactions are proportional to a positive power of $G$, and gravity becomes a free theory for $G=0$. Evidently, then, there is no renormalization for $G=0$, and $G=0$ is a fixed point of the renormalization group. If the bare Newton's constant $G_{0}$ vanishes, then so does the renormalized coupling $G$. We arrive again at the conclusion annunciated earlier; Coleman's mechanism favors the lowest possible value of $G$, and hence is incompatible with observation unless the bare parameter $G_{0}$ is bounded away from zero.

May we also say that a lower bound on the bare Newton's constant $G_{0} \geqslant G_{0, \min }>0$ suffices to ensure that the renormalized $G$ does not vanish? The issue is whether 
$G_{M}^{-1}$ can be infinitely renormalized as $M$ floats from $M_{0}$ down to zero, or, in other words, whether the metric can undergo an infinite field renormalization. Since the renormalization for $M \ll M_{0}$ can be studied perturbatively, and we know that $G_{M}$ stops running, the only question is whether infinite higher-derivative bare couplings can induce an infinite field renormalization at $M \sim M_{0}$. This question is highly nonperturbative and is hard to answer definitively, but I believe that the same screening effects that underlie the inequalities eq. (6.1) will also prevent infinite field renormalization, even if some bare couplings are infinite. The point is that infinite bare couplings run infinitely quickly as the cutoff floats, and so always induce finite effects. Therefore, if the bare Newton's constant is $G_{0} \ll M_{0}^{-2}$, then the renormalized coupling is $G \sim G_{0}$, regardless of the values of the other bare couplings. (For $G_{0} \gtrsim M_{0}^{-2}$, the renormalized coupling is $G \sim M_{0}^{-2}$, in accord with the bound eq. (6.2).)

If the bare coupling $G_{0}$ has a minimum as a function of $\alpha$, one expects on dimensional grounds that the minimum occurs for $G_{0} \sim M_{0}^{-2}$; then the minimal renormalized coupling is also $G \sim M_{0}^{-2}$. This minimal renormalized coupling should coincide with the observed Newton's constant. It is therefore reasonable to identify the wormhole scale $M_{\mathrm{w}}=M_{0}$ with the Planck scale $M_{\mathrm{p}} \sim 10^{19} \mathrm{GeV}$.

While the above discussion suggests that the other bare couplings do not renormalize $G_{0}$ by an enormous factor, each coupling does give a contribution, however small, to the renormalization of $G_{0}$, because all degrees of freedom in the bare theory couple to gravity. In other words, the renormalized coupling $G$ is a function of all the bare parameters. Therefore, the requirement that $G$ attain its minimum value is expected to completely determine the bare theory, and hence to completely determine all low-energy physics. Quantum indeterminacy of the constants of Nature is thus, in principle, avoided.

Of course, this analysis is premised on the assumption that quantum gravity has no nontrivial continuum limit. It is at least a possibility that the renormalization group equations of quantum gravity have a nontrivial fixed point. Then a nontrivial continuum theory could be formulated ${ }^{\star}$. Various dimensionless couplings would be nonvanishing at the fixed pont, and higher-derivative interactions would have important effects in the continuum theory. There would then be a serious danger that these interactions would destabilize the peak at $\Lambda=0$ that Coleman found in the distribution $Q(\alpha)$. But if, as one expects, there is no nontrivial continuum limit of quantum gravity, then our arguments buttress Coleman's prediction that $\Lambda=0$.

To further support these claims, we should now discuss somewhat more explicitly the renormalization group equations of quantum gravity [26]. These equations describe how the effective action $S_{M}$ flows as the floating cutoff $M$ is lowered. The

* The possible existence of a nontrivial fixed point has been discussed (from quite different points of view) by Weinberg [26] and by Antoniadis and Tomboulis [28]. The conclusions of ref. [28] have been criticized by Johnston [29]. 
great utility of these equations derives from the observation that, in the infrared limit $M \rightarrow 0, S_{M}$ exhibits "universal" behavior that depends only rather weakly on the original bare action $S_{M_{0}}$.

If the bare theory $S_{M_{0}}$ depends upon an infinite number of adjustable bare parameters, one might worry that the theory has no predictive power. But as Wilson emphasized [23], physics at the energy scale $M \ll M_{0}$ can be predicted, because, up to corrections suppressed by powers of $M / M_{0}, S_{M}$ can be expressed in terms of a small number of renormalized parameters. Indeed, even the power corrections can be systematically taken into account; to any finite order in $M / M_{0}, S_{M}$ can be expressed in terms of a finite number of renormalized quantities.

In discussing Coleman's mechanism, we consider the value of the renormalized euclidean action at its stationary point. In this context, the expansion of $S_{M}$ in powers of $M$ becomes an expansion of the stationary value of the action in powers of $R^{-1}$, where $R$ is the "radius" of the classical solution. For example, the action $S_{M}[g]$ of eq. (3.5), when evaluated for a four-sphere of radius $R$, becomes

$$
S(R)=\frac{\Omega}{16 \pi G}\left(2 \Lambda R^{4}-12 R^{2}+A_{4}+A_{6} R^{-2}+\ldots\right),
$$

where $\Omega=8 \pi^{2} / 3$ is the volume of a unit four-sphere. Here $A_{4}, A_{6}, \ldots$ arise from terms in the action involving $4,6, \ldots$ derivatives of the metric. Because of renormalization effects, the parameters $A, G, A_{4}, A_{6}, \ldots$, are all implicitly dependent on the radius $R$.

Naively, eq. (6.3) shows "universal" behavior in that $S(R)$ can be expressed to order $R^{-2 m}$ in terms of $m+3$ renormalized parameters. This statement is naive because we have ignored the dependence of the renormalized parameters on $R \sim M^{-1}$. For example, we may wish to claim that $S(R)$ up to order $R^{0}$ can be expressed in terms of only the renormalized $\Lambda$ and $G$. We must argue, then, that as the bare parameters of $S_{M_{0}}$ vary on the surface where $\Lambda_{M}$ and $G_{M}$ have specified values, $A_{4, M}$ varies by an amount of order one (independent of $M$ ). We can analyze this problem in perturbation theory using elementary arguments based on dimensional analysis and power-counting of Feynman diagrams.

(There is a technical point that we should comment on here ${ }^{\star}$. All terms in $S_{M}$ that involve four derivatives of the metric are actually "inessential" [26, 14]; these terms are either topological and can be ignored in perturbation theory or can be eliminated from the action by a local redefinition of the metric $g_{\mu \nu}$. Ordinarily, it is advisable to eliminate inessential couplings when renormalization group equations are derived, but we will find it convenient to consider the renormalization of $A_{4}$. In part, this is because we are ultimately interested in minimizing $S(R)$ with respect to $R$ and the $\alpha$-parameters, subject to an infrared cutoff. The redefinition of the metric

\footnotetext{
* This point was discussed by Grinstein and Wise [15].
} 
that eliminates $A_{4}$ preserves the value of the action at its minimum, but modifies the (invariant) radius of the four-sphere solution. Furthermore, this redefinition depends on $\alpha$; if we change the bare parameters, a change in $A_{4}$ is typically induced, and we must redefine the fields again to eliminate it. Thus, if we were to eliminate $A_{4}$ and then minimize the action with respect to $\alpha$, we would be minimizing subject to an $\alpha$-dependent infrared cutoff. It seems less confusing to keep the infrared cutoff fixed and $\alpha$-independent; we then lose the freedom to eliminate $A_{4}$.)

Roughly speaking, $S_{M}$ can be obtained from $S_{M_{0}}$ in perturbation theory by evaluating Feynman diagrams that are cut off in the ultraviolet at $M_{0}$ and in the infrared at $M$. These diagrams can be divided, according to their sensitivity to the ultraviolet cutoff, into three classes - power-divergent, log-divergent, and convergent.

Power-divergent diagrams are of order $M_{0}^{D}$ where $D>0$ is the superficial degree of divergence of the diagram. These diagrams are "nonuniversal" contributions that are dominated by quantum fluctuations with wave number of order $M_{0}$ and that are sensitive to the detailed form of the bare action $S_{M_{0}}$. Because of the power divergences, the renormalization group flow is very complex for $M \sim M_{0}$; many higher dimension operators in $S_{M}$ contribute to the renormalization of lower dimension operators. But, in perturbation theory, the power-divergent diagrams give a decreasingly important contribution to the flow as $M$ decreases.

Of much greater interest are the log-divergent diagrams that are proportional to a power of $\ln \left(M_{0} / M\right)$. These diagrams receive a significant contribution from quantum fluctuations over a broad range of length scales. Unlike power divergences, the $\log$ divergences are universal; the coefficients of leading and nonleading powers of $\ln \left(M_{0} / M\right)$ are calculable in terms of low-energy physics alone and are insensitive to the details of physics at the cutoff.

Finally, there are convergent diagrams. These too are calculable in terms of low-energy physics alone. Their sensitivity to the cutoff is of order $M_{0}^{D}$, where $D<0$ is the superficial degree of divergence of the diagram. If we are interested in the flow of the renormalized effective action $S_{M}$ for $M \ll M_{0}$, we need consider only the log-divergent and convergent diagrams, for the renormalization effects induced by the power-divergent diagrams are not sensitive to $M$.

Now consider the implications of this classification for the renormalization of quantum gravity. First we consider pure gravity, uncoupled to matter, which we have argued is adequate for studying the asymptotic $M \rightarrow 0$ behavior of the effective action $S_{M}$. Then we will discuss how our conclusions are modified when gravity is coupled to matter with renormalizable or superrenormalizable interactions.

We have argued that the flow of $S_{M}$ for $M$ small is determined by log-divergent and convergent diagrams. But in a theory like pure gravity with $\Lambda=0$, in which all couplings are of nonrenormalizable type, all renormalizations induced by such diagrams are renormalizations of higher dimensional couplings by lower dimen- 


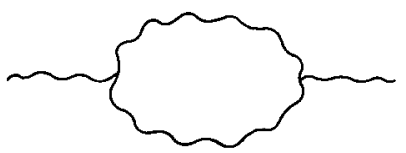

Fig. 15. A one-loop diagram that contributes to the renormalization of the gravitational effective action. All lines represent gravitons.

sional couplings. This conclusion follows directly from simple dimensional analysis. Consider, for example, the logarithmic renormalizations. If we scale $G^{-1}$ out of the action as in eq. (6.3), then $G$ is a loop-counting parameter; an $L$-loop diagram is of order $G^{L-1}$. Since all other couplings have dimensions of mass to a negative power, it follows immediately on dimensional grounds (for $\Lambda=0$ ) that there is no logarithmic renormalization of $G^{-1}$. Similarly, a logarithmic renormalization of $A_{4}$ occurs only in one-loop order, and has the form

$$
\frac{\delta A_{4}}{16 \pi G}=b_{4} \ln \left(M_{0} / M\right)
$$

where $b_{4}$ is a numerical constant. (This renormalization is generated by the diagram in fig. 15, from which the value of $b_{4}$ can be extracted.) Each coupling $A_{2 n}$ receives a logarithmic renormalization only up to $(n-1)$-loop order in perturbation theory [26]. For example, $A_{6}$ receives two-loop renormalization of order $G^{2}$ and a one-loop renormalization of order $G A_{4}$.

We see, therefore, that the infrared-sensitive renormalization of each coupling in the effective action is determined by renormalized couplings of lower dimension. Once we have fixed the value of $\Lambda, G$, and $A_{2 m}$ for $m<n$, the sensitivity of the renormalized $A_{2 n}$ to the bare parameters involves only the nonuniversal powerdivergent renormalizations. In perturbation theory, $G M_{0}^{2}$ and $A_{2 m} M_{0}^{2 m-2}$ are regarded as small, and the power-divergent renormalization of $A_{2 n}$ is bounded by a power of $M_{0}$ determined by dimensional analysis

$$
\left(\delta A_{2 n}\right)_{\text {nonuniversal }} \leq C M_{0}^{-2 n+2}
$$

Our earlier discussion of nonperturbative effects then suggests that an inequality of this form holds even beyond the domain of validity of perturbation theory .

Specifically, the renormalization of the cosmological constant $\Lambda$ is due to power-divergent diagrams only. These diagrams renormalize $\Lambda / 8 \pi G$ by an amount of order $M_{0}^{4}$, and $\Lambda / 8 \pi G$ approaches a finite limit as the floating cutoff $M$ approaches zero. Similarly, in pure gravity with vanishing renormalized $\Lambda, G^{-1}$ is renormalized by power-divergent diagrams only, and it too approaches a finite limit

\footnotetext{
* A related suggestion was made by Weinberg [14].
} 
for $M \rightarrow 0$. We say that $\Lambda$ and $G$ stop running in the far infrared. On the other hand, $A_{4}$ does not stop running; it receives the logarithmic renormalization eq. (6.4). This logarithmic renormalization diverges in the infrared limit $M \rightarrow 0$, but the coefficient of the logarithm is determined by the renormalized $G$. When $\Lambda$ and $G$ are held fixed, then, $S(R)$ in eq. (6.3) varies as a function of the $\alpha$ 's by an amount of order one; it is a bounded function of both $\alpha$ and $R$. Therefore, the $\alpha$-dependence that arises from higher-derivative operators can be neglected in the $R \rightarrow \infty$ limit, just as we assumed in sect. 3 .

The power-counting described above applies to pure gravity, and hence to the renormalization group flow of the gravitational effective action $S_{M}$ in the far infrared, after all matter has been integrated out. It is also of interest to consider the flow of $S_{M}$ at intermediate length scales, where the coupling of gravity to matter cannot be neglected. Because the matter action can contain terms of renormalizable or superrenormalizable type, we must modify the earlier analysis, which applied to a theory in which all interactions are of nonrenormalizable type.

Of particular interest is the dependence of the renormalization of $G$ on the masses of matter particles, and this is the only effect of the matter that we will discuss here in any detail. Insofar as the mass-dependent renormalization of $G$ is calculable, we might hope to reach nontrivial conclusions about the masses of elementary particles form the requirement that $G^{-1}$ attains its largest possible value.

Dimensionally, a calculable order $-m^{2}$ logarithmic renormalization of $G^{-1}$ is possible in one-loop order, and indeed, one finds that this renormalization is generated by the diagram in fig. 16. Calculation of this diagram [30] shows that the logarithmic renormalization is

$$
\delta\left(\frac{1}{16 \pi G}\right)=-\frac{1}{192 \pi^{2}} m^{2} \ln \left(M_{0}^{2} / m^{2}\right)+\mathrm{O}\left(m^{2}\right)+\mathrm{O}\left(G m^{4}\right)
$$

where $m$ is the mass of either a free (Dirac) fermion or of a free minimally coupled (real) scalar. The order- $m^{2}$ correction in eq. (6.6) is a nonuniversal effect (which can be absorbed by shifting $M_{0}^{2}$ in the logarithm), and the order- $G m^{4}$ correction is due to diagrams with two or more loops. Of course, if we include the contributions due to renormalizable interactions, the logarithmic renormalization of $G^{-1}$ is modified. The interactions generate additional logarithms in each order of perturbation theory. These logarithms can be summed, and they have the effect of causing $m^{2}$ to

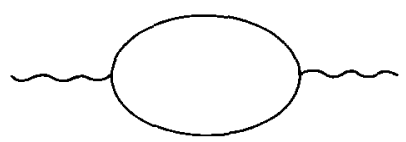

Fig. 16. A one-loop diagram that induces a logarithmic renormalization of $G$. The solid line represents a massive matter particle; it may be either a scalar or a spin $-\frac{1}{2}$ fermion. 
become a scale-dependent running parameter. If the matter particle that induces this renormalization is a composite object (like the pion), then the cutoff $M_{0}$ in the logarithm is not the wormhole scale, but rather the mass scale (like $4 \pi f_{\pi}$ ) below which the particle behaves like an effectively elementary object.

The physical mass $m$ of the matter particle appears in the argument of the logarithm in eq. (6.6). This is because the matter loop continues to contribute to the renormalization of $G^{-1}$ as the cutoff $M$ floats down until $M \sim m$, at which point we integrate the matter particle out of the effective action, and it ceases to contribute to loop diagrams. In spite of the logarithm, though, the renormalization eq. (6.6) actually vanishes in the limit $m^{2} \rightarrow 0$. This observation corroborates our earlier conclusion that $G^{-1}$ stops running in the infrared. Long-wavelength fluctuations of light matter particles, like long-wavelength graviton fluctuations, contribute very little to the renormalization of $G$.

The sign of the calculable logarithmic renormalization in eq. (6.6) is such as to decrease $G^{-1}$. The criterion of maximizing $G^{-1}$, then, favors lighter matter particles. We ought not to accept this conclusion too readily, however. Because the calculable infrared renormalization is small for small $\mathrm{m}^{2}$, one must worry about whether this effect is swamped by nonuniversal contributions.

The form of eq. (6.6) should be contrasted with the logarithmic mass-dependent one-loop renormalization of $A_{4}$; for a minimally coupled real scalar this is

$$
\frac{\delta A_{4}}{16 \pi G}=-\frac{29}{30}\left(\frac{1}{16 \pi^{2}}\right) \ln \left(M_{0}^{2} / m^{2}\right) .
$$

As noted by Grinstein and Wise [15] and Klebanov, Susskind, and Banks [16], then, if the correct criterion to determine the $\alpha$ 's were to minimize $A_{4}$ rather than to maximize $G^{-1}$, then eq. (6.7) would favor that $m^{2}$ approach zero. But furthermore, because the mass-dependent renormalization of $A_{4}$ becomes infinite for $m^{2} \rightarrow 0$, we can be confident that the calculable dependence of $A_{4}$ on the physical mass $\mathrm{m}^{2}$ dominates nonuniversal effects for $m^{2} \rightarrow 0$. We would conclude, therefore, that the pion mass $m_{\pi}$ is exactly zero, if $m_{\pi}=0$ can be achieved for any value of the $\alpha$ 's. (It does not even matter that, for the pion, $M_{0}$ in eq. (6.7) is of order $4 \pi f_{\pi}$, rather than the wormhole scale.)

This unpleasant conclusion can be avoided if, as we have proposed, the correct criterion to determine the $\alpha$ 's is actually that $G^{-1}$ is at is maximum. Then bare parameters at the wormhole scale must be adjusted so as to maximize the sum of the calculable infrared renormalization of $G^{-1}$ in eq. (6.5) and of the uncalculable (but non-negligible) nonuniversal contributions to the renormalization of $G^{-1}$. The nature of the nonuniversal contributions depends on whether the light particles have bare masses or acquire their masses from renormalizable interactions.

Consider, for example, an elementary scalar with an adjustable bare mass. In this case we can argue that if the bare mass $m_{0}$ is chosen to maximize $G^{-1}$, then the 
renormalized scalar mass must either vanish or be of the order of the cutoff $M_{0}$. If $G^{-1}$ is stationary as a function of $m_{0}^{2}$, we must have

$$
\begin{aligned}
0 & =\frac{\partial}{\partial m_{0}^{2}}(16 \pi G)^{-1} \\
& =\frac{\partial}{\partial m_{0}^{2}}\left(16 \pi G_{0}\right)^{-1}-\frac{1}{192 \pi^{2}} \ln \left(M_{0}^{2} / m^{2}\right)\left(\frac{\partial}{\partial m_{0}^{2}} m^{2}\right)+\ldots
\end{aligned}
$$

where $G_{0}$ includes all nonuniversal mass-dependent contributions to the renormalization of $G$. But because we expect that

$$
\begin{gathered}
\frac{\partial}{\partial m_{0}^{2}} m^{2} \sim 1, \\
\frac{\partial}{\partial m_{0}^{2}}\left(16 \pi G_{0}\right)^{-1} \lesssim \frac{1}{192 \pi^{2}},
\end{gathered}
$$

eq. (6.8) can be satisfied only for

$$
\ln \left(M_{0}^{2} / m^{2}\right) \sim 1
$$

Thus, while $16 \pi G_{0}^{-1}$ might be a complicated function of $m_{0}^{2}$ that is maximized at a nontrivial value of $m_{0}^{2}$, this maximum will be destabilized by the calculable infrared-sensitive renormalization of $G$, if $m^{2} \ll M_{0}^{2}$.

The situation is quite different, however, in the more realistic case of a particle that has no bare mass and acquires its physical mass from a renormalizable interaction, like a Yukawa coupling. In this case, we require $G^{-1}$ to be stationary as a function of the bare Yukawa coupling $\lambda_{0}$, or

$$
0=\frac{\partial}{\partial \lambda_{0}^{2}}\left(16 \pi G_{0}\right)^{-1}-\frac{1}{192 \pi^{2}} \ln \left(M_{0}^{2} / m^{2}\right)\left(\frac{\partial}{\partial \lambda_{0}^{2}} m^{2}\right)+\ldots
$$

But now we expect

$$
\frac{\partial}{\partial \lambda_{0}^{2}}\left(16 \pi G_{0}\right)^{-1} \leq \frac{1}{\left(16 \pi^{2}\right)^{2}} M_{0}^{2}
$$

due to diagrams like fig. 17 , and

$$
\frac{\partial}{\partial \lambda_{0}^{2}} m^{2} \sim v^{2}
$$




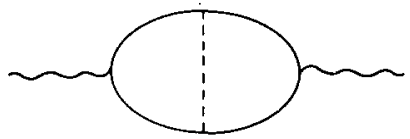

Fig. 17. A two-loop diagram that contributes to the renormalization $G$. The solid line represents a spin $-\frac{1}{2}$ fermion, and the dotted line represents a scalar.

where $v$ is an expectation value that is determined by other, independent bare parameters ${ }^{\star}$. The maximum of $G_{0}^{-1}$ will not be destabilized by the infrared renormalization of $G$, then, provided only that $v^{2} \ll M_{0}^{2}$.

We see, then, that if one accepts Coleman's explanation for the vanishing of the cosmological constant, then one must also find unacceptable any elementary particle that is light compared to the wormhole scale and that has a bare mass that can be adjusted at the wormhole scale, unless the physical mass of the particle vanishes identically $\star \star$. This argument has nothing to do with the quadratically divergent renormalization of the mass of an elementary scalar. In fact, the same conclusion applies to a light elementary fermion with an adjustable bare mass, even though the fermion mass is protected from any power-divergent renormalization by approximate chiral symmetry.

If Coleman's mechanism is to apply to Nature, we must require therefore that all mass scales of low-energy physics are determined dynamically, rather than by adjusting bare mass parameters in the action $S_{M_{0}}$ at the wormhole scale. This conclusion is actually unsurprising. If the bare parameters are to be determined by the infrared-insensitive renormalization of $G$, it would evidently require a miracle for the bare Higgs mass, for example, to be fine-tuned just so as to ensure that the weak-interaction mass scale is far below the Planck scale. But if all low-energy mass scales are determined dynamically, then it seems at least conceivable that the low-energy physics that we observe can be accounted for (to excellent accuracy) by maximizing the infrared-insensitive nonuniversal renormalization of $G^{-1}$.

The curious and rather surprising thing that we have found is that a light elementary particle with an adjustable bare mass is compatible with Coleman's mechanism if its physical mass is exactly zero. This observation, unfortunately, does not help us to understand why the weak-interaction scale is so small compared to the Planck scale, but it might have other interesting implications for low-energy physics.

In the end, we have been unable to extract from wormhole physics any quantitative conclusion other than $\Lambda=0$. Indeed, it seems unlikely that any other precise

\footnotetext{
* The logarithmic renormalization of $\lambda$ has been ignored here.

* I am assuming that the $\alpha$-dependence of the bare mass is such that the physical mass vanishes for some value of $\alpha$. Note also that, in order to simplify the discussion, I have assumed that elementary scalars are minimally coupled.
} 
predictions can be made without a detailed understanding of Planck-scale physics. This is disappointing. On the other hand, we have probed carefully for fatal flaws in Coleman's beautiful explanation for the vanishing of the cosmological constant, and have found none. This is encouraging.

This research began in collaboration with Andy Cohen and Mark Wise; I am very grateful to both of them for many enlightening discussions. I have also benefited from conversations about wormholes with Jim Cline, Sidney Coleman, Gerry Gilbert, Stephen Hawking, Igor Klebanov, and Frank Wilczek.

\section{Notes Added}

There is an interesting exception to the general feature that the dependence of the renormalized Newton's constant $G$ on other renormalized parameters cannot be computed. The dependence of $G$ on the vacuum angle $\theta$ of quantum chromodynamics is determined by low-energy physics [31]. This exception arises because the $\theta$-dependence of $G$ is entirely due to nonperturbative strong-interaction effects that are extremely weak at very short distances.

More careful consideration indicates that the excluded volume interaction between large and small instantons, discussed in sect. 5, does not by itself suffice to suppress the effects of large wormholes. Rather, these interactions induce a very strong renormalization of the relation between the $\alpha$-parameters of the large wormholes and the density of large instantons. I am indebted to Joe Polchinski for an enlightening discussion of this point. (See ref. [32].) If the large wormhole problem is to be evaded within the framework described in this paper, it will presumably be necessary to invoke a more subtle interaction between large and small instantons. Other recent discussions of the large wormhole problem may be found in ref. [33].

\section{References}

[1] S.W. Hawking, Phys. Lett. B195 (1987) 337: Phys. Rev. D37 (1988) 904

[2] G.V. Lavrelashvili, V. Rubakov and P.G. Tinyakov, JETP Lett. 46 (1987) 167

[3] S.B. Giddings and A. Strominger, Nucl. Phys. B306 (1988) 890;

K. Lee, Phys. Rev. Lett. 61 (1988) 263

[4] S.W. Hawking, in Relativity, groups, and topology II, ed. B. DeWitt and R. Stora (North-Holland, Amsterdam, 1983)

[5] J. Hartle, in High energy physics 1985, ed. M. Bowick and F. Gürsey (World Scientific, Singapore, 1986)

[6] J. Hartle and S.W. Hawking, Phys. Rev. D28 (1983) 2960

[7] S.W. Hawking, D.N. Page and C.N. Pope, Nucl. Phys. B170 (1980) 283;

S.W. Hawking, Commun. Math. Phys. 87 (1982) 395; Nucl. Phys. B244 (1984) 135

[8] S. Coleman, Nucl. Phys. B307 (1988) 867

[9] S.B. Giddings and A. Strominger, Nucl. Phys. B307 (1988) 854

[10] S. Coleman, Nucl. Phys. B310 (1988) 643 
[11] T. Banks, Nucl. Phys. B309 (1988) 493

[12] S.W. Hawking and R. Laflamme, Phys. Lett. B209 (1988) 39

[13] S.B. Giddings and A. Strominger, Baby universes, third quantization, and the cosmological constant, Harvard University preprint HUTP-88/A036 (1988)

[14] S. Weinberg, Rev. Mod. Phys. 61 (1989) 1

[15] B. Grinstein and M.B. Wise, Phys. Lett. B212 (1988) 407

[16] I. Klebanov, L. Susskind and T. Banks, Wormholes and the cosmological constant, SLAC preprint SLAC-PUB-4705 (1988);

I. Klebanov and L. Susskind, Wormholes and cosmology, SLAC preprint SLAC-PUB-4734 (1988)

[17] A. Gupta and M.B. Wise, Comment on wormhole correlations (Revised Version), Caltech preprint CALT-68-1520 (1988)

[18] W. Fischler and L. Susskind, Phys. Lett. B217 (1989) 48

[19] M. Srednicki, Infinite quantization, UC Santa Barbara preprint UCSBTH-88-07 (1988);

S. Adler, On the Banks-Coleman-Hawking argument for the vanishing of the cosmological constant, Institute for Advanced Study preprint IASSNS-HEP 88/35 (1988);

J. Kim and K. Lee, The Scale problem in wormhole physics, FNAL preprint FERMILAB-PUB88/95-T (1988);

M.B. Mijić, On the probability of having a universe with small cosmological constant, Santa Barbara Institute for Theoretical Physics preprint NSF-ITP-88-128 (1988);

G. Gilbert, Nucl. Phys. B, to be published;

R.C. Myers and V. Periwal, Constraints and correlations in the Coleman calculus, Santa Barbara Institute for Theoretical Physics preprint NSF-ITP-88-151 (1988)

[20] V. Kaplanovsky, unpublished

[21] S.W. Hawking, Phys. Lett. B134 (1984) 403

[22] E. Baum, Phys. Lett. B133 (1983) 185

[23] K. Wilson and J. Kogut, Phys. Rep. 12 (1974) 75

[24] R.P. Feynman, Statistical mechanics (W.A. Benjamin, New York, 1972)

[25] C.G. Callan, R. Dashen and D.J. Gross, Phys. Rev. D17 (1978) 2717

[26] S. Weinberg, in General relativity: an Einstein centenary survey, ed. S.W. Hawking and W. Israel (Cambridge University Press, Cambridge, 1979)

[27] M. Aizenman, Phys. Rev. Lett. 47 (1981); Commun. Math. Phys. 86 (1982) 1;

J. Fröhlich, Nucl. Phys. B200 (1982) 281

[28] I. Antoniadis and E.T. Tomboulis, Phys. Rev. D33 (1986) 2756

[29] D. Johnston, Nucl. Phys. B297 (1988) 721

[30] P. Candelas and D.J. Raine, Phys. Rev. D12 (1975) 965

[31] H. B. Nielsen and M. Ninomiya, A solution to the strong CP problem in baby universe theory. INS-721 (1988);

J. Preskill, S. T. Trivedi and M. B. Wise, Wormholes in spacetime and $\theta_{\mathrm{QCD}}$, Caltech preprint CALT-68-1539 (1989);

K. Choi and R. Holman, A wormhole solution to the strong CP problem, Carnegie-Mellon preprint CMU-HEP89-04 (1989)

[32] J. Polchinski, Decoupling versus excluded volume or return of the giant wormholes, University of Texas preprint UTTG-06-89 (1989)

[33] B. Grinstein, Charge quantization of wormholes and the finiteness of Newton's constant, FERMILAB-PUB-88/210-T (1988);

$\mathrm{S}$. Coleman and K. Lee, Escape from the menace of the giant wormholes, Harvard preprint HUTP-89/A002 (1989);

S.-J. Rey, The collective dynamics and the correlations of wormholes in quantum gravity, UCSB print-89-0096 (1989);

R. Brustein and S. P. deAlwis, The cluster expansion for wormholes, UTTG-04-89 (1989) 